\title{
Uniqueness of bubbling solutions with collapsing singularities
}

\author{
Youngae Lee ${ }^{\mathrm{a}, *}$, Chang-Shou Lin ${ }^{\mathrm{b}}$ \\ a Department of Mathematics Education, Teachers College, Kyungpook National \\ University, Daegu, South Korea \\ b Taida Institute for Mathematical Sciences, Center for Advanced Study in \\ Theoretical Sciences, National Taiwan University, Taipei 106, Taiwan
}

\section{A R T I C L E I N F O}

\section{Article history:}

Received 12 July 2018

Accepted 5 February 2019

Available online 13 February 2019

Communicated by H. Brezis

\section{$M S C$ :}

$35 \mathrm{~A} 05$

$53 \mathrm{~A} 30$

$35 \mathrm{~J} 15$

58J05

Keywords:

Mean field equation

Bubbling solutions

Uniqueness

Collapse of singularities
A B S T R A C T

The seminal work [7] by Brezis and Merle showed that the bubbling solutions of the mean field equation have the property of mass concentration. Recently, Lin and Tarantello in [30] found that the "bubbling implies mass concentration" phenomena might not hold if there is a collapse of singularities. Furthermore, a sharp estimate [23] for the bubbling solutions has been obtained. In this paper, we prove that there exists at most one sequence of bubbling solutions if the collapsing singularity occurs. The main difficulty comes from that after re-scaling, the difference of two solutions locally converges to an element in the kernel space of the linearized operator. It is well-known that the kernel space is three dimensional. So the main technical ingredient of the proof is to show that the limit after re-scaling is orthogonal to the kernel space.

() 2019 Elsevier Inc. All rights reserved.

\footnotetext{
* Corresponding author.

E-mail addresses: youngaelee@knu.ac.kr (Y. Lee), cslin@math.ntu.edu.tw (C.-S. Lin).
} 


\section{Introduction}

We are concerned with the following mean field type equation:

$$
\left\{\begin{array}{l}
\Delta_{M} u+\rho\left(\frac{h_{*} e^{u}}{\int_{M} h_{*} e^{u} d v_{g}}-1\right)=4 \pi \sum_{q_{i} \in S} \alpha_{i}\left(\delta_{q_{i}}-1\right) \text { in } M \\
\int_{M} u d v_{g}=0
\end{array}\right.
$$

where $(M, d s)$ is a compact Riemann surface, $\rho>0, d v_{g}$ is the volume form, $\Delta_{M}$ is the Laplace-Beltrami operator on $(M, d s), S \subseteq M$ is a finite set of distinct points $q_{i}$, $\alpha_{q_{i}}>-1$, and $\delta_{q_{i}}$ is the Dirac measure at $q_{i}$. The point $q_{i}$ with Dirac measure is called vortex point or singular source. Throughout this paper, we always assume that $|M|=1, h_{*}>0$ and $h_{*} \in C^{3}(M)$. The equation (1.1) arises in various different fields. In conformal geometry, (1.1) is related to the Nirenberg problem of finding prescribed Gaussian curvature if $S=\varnothing$, and the existence of a positive constant curvature metric with conic singularities if $S \neq \varnothing$ (see [42] and the references therein). The equation (1.1) is also related to the self-dual equation of the relativistic Chern-Simons-Higgs model. For the recent developments related to (1.1), we refer to the readers to $[6,4,2,7,15,11-14$, 29,33,31,32,35-37,39,42,44,43,5,8,17-21,28,38,41,45] and references therein.

In the seminal work [7] by Brezis and Merle, the blow up behavior of solutions for (1.1) has been studied as follows:

Theorem A. [7,27,4] Given fixed each vortex point $q_{i} \in S$, suppose $\alpha_{i} \in \mathbb{N}, i=1, \cdots, N$. We assume that $h^{*}$ is a positive smooth function. Let $u_{k}^{*}$ be a sequence of blow up solutions for (1.1), that is: $\max _{M} u_{k}^{*} \rightarrow+\infty$ as $k \rightarrow+\infty$. Then there is a non-empty finite set $\mathcal{B}$ (blow up set) such that,

$$
\rho \frac{h^{*} e^{u_{k}^{*}}}{\int_{M} h^{*} e^{u_{k}^{*}} d v_{g}} \rightarrow \sum_{p \in \mathcal{B}} \beta_{p} \delta_{p}, \text { where } \beta_{p} \in 8 \pi \mathbb{N}
$$

For equation (1.1), we call $\rho \frac{h^{*} e^{u^{*}}}{\int_{M} h^{*} e^{u^{*}} d v_{g}}$ the mass distribution of the solution $u^{*}$. Following this terminology, Theorem A states that: When the vortex points are not collapsing, the mean field equation possesses the property of the so-called "blow up solutions has the mass concentration property". The version of Theorem A for the following Chern-Simons-Higgs (CSH) equation was also proved in [15, Theorem 3.1]:

$$
\Delta_{\mathbb{T}} u+\frac{1}{\varepsilon^{2}} e^{u}\left(1-e^{u}\right)=4 \pi \sum_{j=1}^{N} \delta_{p_{j}} \text { in } \mathbb{T},
$$

where $\varepsilon>0$ is a small parameter and $\mathbb{T}$ is a flat torus. The equation (1.2) was derived from the $\mathrm{CSH}$ model to describe vortices in high temperature superconductivity, and has been extensively studied during few decades. We refer the readers to $[22,40,44,15$, 16,33,34,39] and references therein. Among them, Lin and Yan in [34] proved the local 
uniqueness result of bubbling solutions for (1.2), that is, if $u_{\varepsilon, 1}$ and $u_{\varepsilon, 2}$ are two sequence of bubbling solutions blowing up at the same points under some non-degenerate condition, then $u_{\varepsilon, 1}=u_{2, \varepsilon}$ for small $\varepsilon>0$. By applying the idea in [34], the local uniqueness result of bubbling solutions of (1.1) was obtained in [3]. We note that the works [34,3] are concerned with the local uniqueness of bubbling solutions when the vortex points are not collapsing.

However, there is a big difference when the collapsing singularities are considered. First, Lin and Tarantello in [30] observed a new phenomena such that blow-up solutions with collapsing singularities might not have the "concentration" property of its mass distribution. The general version was studied in [23]. To describe the results, let us consider the following equation:

$$
\left\{\begin{array}{l}
\Delta_{M} u_{t}^{*}+\rho\left(\frac{h^{*} e^{u_{t}^{*}}}{\int_{M} h^{*} e^{u_{t}^{*}} d v_{g}}-1\right) \\
=4 \pi \sum_{i=1}^{d} \alpha_{i}\left(\delta_{q_{i}(t)}-1\right)+4 \pi \sum_{i=d+1}^{N} \alpha_{i}\left(\delta_{q_{i}}-1\right) \text { in } M, \\
\int_{M} u_{t}^{*} d v_{g}=0
\end{array}\right.
$$

where $\lim _{t \rightarrow 0} q_{i}(t)=\mathfrak{q} \notin\left\{q_{d+1}, \cdots, q_{N}\right\}, \forall i=1, \cdots, d$ and $q_{i}(t) \neq q_{j}(t)$ for $i \neq j \in$ $\{1, \cdots, d\}$. Then the following holds:

Theorem B. $[30,23]$ Assume $\alpha_{i} \in \mathbb{N}, 1 \leq i \leq N$. Let $u_{t}^{*}$ be a sequence of blow up solutions of (1.3) with $\rho \notin 8 \pi \mathbb{N}$. Then $u_{t}^{*}$ blows up only at $\mathfrak{q} \in M$. Furthermore, there exists a function $w^{*}$ such that

$$
u_{t}^{*} \rightarrow w^{*} \text { in } C_{\mathrm{loc}}^{2}(M \backslash\{\mathfrak{q}\})
$$

as $t \rightarrow 0$, and $w^{*}$ satisfies:

$$
\left\{\begin{array}{l}
\Delta_{M} w^{*}+(\rho-8 m \pi)\left(\frac{h^{*} e^{w^{*}}}{\int_{M} h^{*} e^{w^{*}}}-1\right) \\
=4 \pi\left(\sum_{i=1}^{d} \alpha_{i}-2 m\right)\left(\delta_{q}-1\right)+4 \pi \sum_{i=d+1}^{N} \alpha_{i}\left(\delta_{q_{i}}-1\right) \text { in } M, \\
\int_{M} w^{*} d v_{g}=0,
\end{array}\right.
$$

for some $m \in \mathbb{N}$ with $1 \leq m \leq\left[\frac{1}{2} \sum_{i=1}^{d} \alpha_{i}\right]^{1}$ and $\rho>8 m \pi$.

So Theorem B tells us that the mass concentration does no longer hold if the collapsing singularity occurs. Indeed, we have $\lim _{t \rightarrow 0} \int_{M} h^{*} e^{u_{t}^{*}} d v_{g}<+\infty$, which is different from the situation described in Theorem A. We note that Theorem B could be improved provided that the following nondegeneracy condition holds:

\footnotetext{
$1[x]$ stands for the integer part of $x$.
} 
Definition 1.1. A solution $w^{*}$ of (1.4) is said non-degenerate, if the linearized problem

$$
\Delta_{M} \phi+(\rho-8 m \pi) \frac{h^{*} e^{w^{*}}}{\int_{M} h^{*} e^{w^{*}} d v_{g}}\left(\phi-\frac{\int_{M} h^{*} e^{w^{*}} \phi d v_{g}}{\int_{M} h^{*} e^{w^{*}} d v_{g}}\right)=0, \int_{M} \phi d v_{g}=0
$$

only admits the trivial solution.

Using the transversality theorem, we can always choose a positive smooth function $h^{*}$ such that $w^{*}$ is non-degenerate. See Theorem 4.1 in [24]. Based on the non-degeneracy assumption for (1.4), some sharper estimates on the bubbling solutions of (1.3) were obtained in [23] (see also section 2 below).

For the simplicity, throughout this paper, we focus on the case where the collapsing vortices are only two, that is,

$$
d=2, \alpha_{1}=\alpha_{2}=1, \alpha_{i} \in \mathbb{N} \text { for } i=3, \cdots, N
$$

Our main goal is to prove the local uniqueness of blow up solutions of (1.3) with collapsing singularities.

Theorem 1.2. Assume that (1.6) holds and $\rho \notin 8 \pi \mathbb{N}$. Suppose that $u_{t, 1}^{*}$ and $u_{t, 2}^{*}$ are two blow up solutions of (1.3). Assume that $u_{t, 1}^{*}, u_{t, 2}^{*} \rightarrow w^{*}$ in $C_{l o c}(M \backslash\{\mathfrak{q}\})$, where $w^{*}$ is a non-degenerate solution of (1.4) with $m=1$. Then $u_{t, 1}^{*}=u_{t, 2}^{*}$ for sufficiently small $t>0$.

We remark that the study of blow up solutions of (1.3) with collapsing singularities is also important to compute the topological degree for the Toda system as noticed in $[24,26]$, where the degree counting of the whole system is reduced to computing the degree of the corresponding shadow system (see [24, Theorem 1.4]). Thus it is inevitable to encounter with the phenomena of collapsing singularities when we want to find a priori bound for solutions of an associated shadow system. For the details, we refer to the readers to $[24,26]$.

In order to prove Theorem 1.2, we need to analyze the asymptotic behaviour of $\zeta_{t}=$ $\frac{u_{t, *}^{(1)}-u_{t, *}^{(2)}}{\left\|u_{t, *}^{(1)}-u_{t, *}^{(2)}\right\|_{L^{\infty}(M)}}$. After a suitable scaling on small domain of $\mathfrak{q}, \zeta_{t}$ converges to an entire solution of the linearized problem associated to the Liouville equation:

$$
\Delta v+e^{v}=0 \text { in } \mathbb{R}^{2},
$$

where $\Delta=\sum_{i=1}^{2} \frac{\partial^{2}}{\partial x_{i}^{2}}$ denotes the standard Laplacian in $\mathbb{R}^{2}$. A solution $v$ of (1.7) is completely classified [10] such that

$$
v(z)=v_{\mu, a}(z)=\ln \frac{8 e^{\mu}}{\left(1+e^{\mu}|z+a|^{2}\right)^{2}}, \quad \mu \in \mathbb{R}, a=\left(a_{1}, a_{2}\right) \in \mathbb{R}^{2} .
$$


The freedom in the choice of $\mu$ and $a$ is due to the invariance of equation (1.7) under dilations and translations. The linearized operator $L$ relative to $v_{0,0}$ is defined by,

$$
L \phi:=\Delta \phi+\frac{8}{\left(1+|z|^{2}\right)^{2}} \phi \quad \text { in } \mathbb{R}^{2} .
$$

In [1, Proposition 1], it has been proved that any kernel of $L$ is a linear combination of $Y_{0}, Y_{1}, Y_{2}$, where

$$
\left\{\begin{array}{l}
Y_{0}(z):=\frac{1-|z|^{2}}{1+|z|^{2}}=-1+\frac{2}{1+|z|^{2}}=\left.\frac{\partial v_{\mu, a}}{\partial \mu}\right|_{(\mu, a)=(0,0)} \\
Y_{1}(z):=\frac{z_{1}}{1+|z|^{2}}=-\left.\frac{1}{4} \frac{\partial v_{\mu, a}}{\partial a_{1}}\right|_{(\mu, a)=(0,0)} \\
Y_{2}(z):=\frac{z_{2}}{1+|z|^{2}}=-\left.\frac{1}{4} \frac{\partial v_{\mu, a}}{\partial a_{2}}\right|_{(\mu, a)=(0,0)}
\end{array}\right.
$$

The orthogonality to $Y_{1}, Y_{2}$ can be obtained by applying a suitable Pohozaev-type identities as in [34]. However, due to the non-concentration of mass, we meet a new difficulty to show the orthogonality with $Y_{0}$. In order to overcome this obstacle, we apply the Green's representation formula with some delicate analysis. This idea comes from the recent work [25]. In [25], it was proved that if $w^{*}$ is a non-degenerate solution of (1.4), and the assumptions (1.6) and $\rho \notin 8 \pi \mathbb{N}$ hold, then there is a blow up solution $u_{t}^{*}$ of (1.3) such that $u_{t}^{*} \rightarrow w^{*}$ in $C_{\text {loc }}(M \backslash\{\mathfrak{q}\})$.

This paper is organized as follows. In section 2, we review some known sharp estimates for blow up solutions of (1.3). In section 3, we analyze the limit behavior of $\zeta_{t}$ in $M \backslash\{\mathfrak{q}\}$ and a small neighborhood of $\mathfrak{q}$ respectively. Finally, we prove Theorem 1.2 by using suitable Pohozaev-type identities and Green's representation formula.

\section{Preliminary}

Let $G(x, p)$ denote the Green's function for the Laplace Beltrami operator $\Delta_{M}$ on $M$, that is

$$
\Delta_{M} G(x, p)+\left(\delta_{p}-1\right)=0, \quad \int_{M} G(x, p) d \sigma(x)=0 .
$$

We recall the following assumption:

$$
d=2, \alpha_{1}=\alpha_{2}=1, \alpha_{i} \in \mathbb{N} \text { for } i=3, \cdots, N
$$

Let $u_{t}^{*}$ be a sequence of blow up solutions of (1.3) and $w^{*}$ be the limit of $u_{t}^{*}$ in Theorem B. Set

$$
u_{t}(x)=u_{t}^{*}(x)+4 \pi \sum_{i=1}^{2} G\left(x, q_{i}(t)\right)+4 \pi \sum_{i=3}^{N} \alpha_{i} G\left(x, q_{i}\right)
$$


and

$$
w(x)=w^{*}(x)+4 \pi \sum_{i=3}^{N} \alpha_{i} G\left(x, q_{i}\right) .
$$

We may choose a suitable coordinate centered at $\mathfrak{q}$ and

$$
\mathfrak{q}=0, \quad q_{1}(t)=t \underline{e}, \quad q_{2}(t)=-t \underline{e}, \quad \text { where } \underline{e} \quad \text { is a fixed unit vector in } \mathbb{S}^{1}
$$

We can rewrite equation (1.3) as follows

$$
\left\{\begin{array}{l}
\Delta_{M} u_{t}+\rho\left(\frac{h(x) e^{u_{t}(x)-G_{t}(x)}}{\int_{M} h e^{u_{t}-G_{t}} d v_{g}}-1\right)=0 \\
\int_{M} u_{t} d v_{g}=0
\end{array}\right.
$$

where

$$
\begin{aligned}
& G_{t}(x):=4 \pi G(x, \underline{t} \underline{e})+4 \pi G(x,-t \underline{e}), \text { and } \\
& h(x):=h_{*}(x) \exp \left(-4 \pi \sum_{i=3}^{N} \alpha_{i} G\left(x, q_{i}\right)\right) \geq 0, \quad h \in C^{3}(M), \quad h(0)>0 .
\end{aligned}
$$

From Theorem B, we have that $u_{t}(x) \rightarrow w(x)+8 \pi G(x, 0)$ in $C_{\text {loc }}^{2}(M \backslash\{0\})$ and $w$ satisfies

$$
\left\{\begin{array}{l}
\Delta_{M} w+(\rho-8 \pi)\left(\frac{h(x) e^{w(x)}}{\int_{M} h e^{w} d v_{g}}-1\right)=0 \\
\int_{M} w d v_{g}=0, \quad w \in C^{2}(M)
\end{array}\right.
$$

We assume that the local isothermal coordinate system satisfies

$$
d s^{2}=e^{2 \varphi(x)}|d x|^{2}, \varphi(0)=\nabla \varphi(0)=0,
$$

that is, $e^{2 \varphi} \Delta_{M}=\Delta$, where $\Delta=\sum_{i=1}^{2} \frac{\partial^{2}}{\partial x_{i}^{2}}$ denotes the standard Laplacian in $\mathbb{R}^{2}$. Fix a small constant $r_{0} \in\left(0, \frac{1}{2}\right)$. It is well known that the conformal factor $\varphi$ is a solution of

$$
-\Delta \varphi=e^{2 \varphi} K \text { in } B_{r_{0}}(0)
$$

where $K(p)$ is the Gaussian curvature at $p \in M$.

Let $\bar{\varphi}(x)$ satisfy the following local problem:

$$
\Delta \bar{\varphi}-e^{2 \varphi} \rho=0 \quad \text { in } \quad B_{r_{0}}(0), \quad \bar{\varphi}(0)=\nabla \bar{\varphi}(0)=0 .
$$

We denote

$$
\psi=2 \varphi+\bar{\varphi}
$$


In view of (2.8) and (2.10), we note that

$$
\begin{gathered}
\nabla_{x} \psi(x)=\nabla \psi(0)+O(|x|)=O(|x|), \nabla_{x} \bar{\varphi}(x)=O(|x|), \\
\nabla_{x} \varphi(x)=O(|x|) \text { for } x \in B_{r_{0}}(0) .
\end{gathered}
$$

By using the local coordinate, we also set the regular part of Green function $G\left(x, q_{i}(t)\right)$ to be

$$
R\left(x, q_{i}(t)\right)=G\left(x, q_{i}(t)\right)+\frac{1}{2 \pi} \ln \left|x-q_{i}(t)\right| .
$$

Let

$$
R_{t}(x):=4 \pi R(x, \underline{t} \underline{)})+4 \pi R(x,-t \underline{e}) .
$$

Therefore we can formulate the local version of (2.4) around 0 as follows:

$$
\Delta \bar{u}_{t}+h_{1}(x)|x-t \underline{e}|^{2}|x+t \underline{e}|^{2} e^{\bar{u}_{t}(x)}=0 \text { in } B_{r_{0}}(0),
$$

where

$$
\begin{aligned}
& \bar{u}_{t}(x)=u_{t}(x)-\ln \int_{M} h e^{u_{t}-G_{t}} d v_{g}-\bar{\varphi}(x), \\
& h_{1}(x)=\rho h(x) e^{\psi(x)-R_{t}(x)}, \quad h_{1}(x)>0 \text { in } B_{r_{0}}(0) .
\end{aligned}
$$

In order to study the behaviour of $\bar{u}_{t}$ near the origin, we consider the scaled sequence

$$
v_{t}(y)=\bar{u}_{t}(t y)+6 \ln t, x \in B_{\frac{r_{0}}{t}}(0),
$$

which satisfies:

$$
\left\{\begin{array}{l}
\Delta v_{t}+h_{t}(y) e^{v_{t}(y)}=0 \text { in } B_{\frac{r_{0}}{t}}(0) \\
\int_{B_{\frac{r_{0}}{t}}(0)} h_{t}(y) e^{v_{t}(y)} d y \leq C
\end{array}\right.
$$

with

$$
h_{t}(y)=h_{1}(t y)|y-\underline{e}|^{2}|y+\underline{e}|^{2}=\rho h(t y) e^{\psi(t y)-R_{t}(t y)}|y-\underline{e}|^{2}|y+\underline{e}|^{2} .
$$

In [23], the following result was obtained.

Theorem C. [23, Theorem 1.2, Section 5] Assume that (1.6) holds and $\rho \notin 8 \pi \mathbb{N}$. Suppose that $u_{t}$ be a sequence of blow up solutions of (2.4). Then the scaled function $v_{t}$ defined by (2.17) blows up at 0 . 
Now we are going to give refined estimates than those provided in Theorem B and Theorem $\mathrm{C}$ under the non-degeneracy assumption for (2.7). To state our result, we fix a constant $R_{0}>2$, and define the following notations:

$$
\begin{aligned}
& \lambda_{t}=\max _{B_{r_{0}}(0)} v_{t}=v_{t}\left(p_{t}\right), \\
& \rho_{t}=\frac{\int_{B_{t R_{0}}\left(t p_{t}\right)} \rho h e^{u_{t}-G_{t}^{(2)}} d v_{g}}{\int_{M} h e^{u_{t}-G_{t}^{(2)}} d v_{g}}, \\
& C_{t}=\frac{1}{8} h_{1}\left(t p_{t}\right)\left|p_{t}-\underline{e}\right|^{2}\left|p_{t}+\underline{e}\right|^{2}, \\
& \widetilde{\phi}_{t}(x)=u_{t}(x)-w(x)-\rho_{t} G\left(x, t p_{t}\right) .
\end{aligned}
$$

Let $\left\|\widetilde{\phi}_{t}\right\|_{*}=\left\|\widetilde{\phi}_{t}\right\|_{C^{1}\left(M \backslash B_{2 R_{0} t}\left(t p_{t}\right)\right)}$. Then we have the following result.

Theorem D. [23, Theorem 1.4, Section 5] Assume that (1.6) holds and $\rho \notin 8 \pi \mathbb{N}$. Let $u_{t}$ be the sequence of blow up solutions of (2.4) and $w+8 \pi G(x, 0)$ be its limit in $M \backslash\{0\}$. If $w$ is a non-degenerate solution of (2.7), then

(i) $\left\|\widetilde{\phi}_{t}\right\|_{*}=O(t \ln t)$,

(ii)

$$
\begin{aligned}
& \lambda_{t}+2 \ln t-\ln \left(\frac{\rho}{\rho-8 \pi} \int_{M} h e^{w}\right)+w\left(t p_{t}\right)+2 \ln C_{t}+8 \pi R\left(t p_{t}, t p_{t}\right) \\
& =O(t \ln t),
\end{aligned}
$$

(iii) $\rho_{t}-8 \pi=O\left(t^{2} \ln t\right)$,

(iv) $\left|\int_{M} h e^{u_{t}-G_{t}} d v_{g}-\frac{\rho}{\rho-8 \pi} \int_{M} h e^{w} d v_{g}\right|=O(t)$,

(v) $\left|p_{t}\right|=O(t)$.

In order to prove Theorem D, the authors in [23] analyzed the scaled function $v_{t}$ with the following ingredients: Set

$$
I_{t}(y)=\ln \frac{e^{\lambda_{t}}}{\left(1+C_{t} e^{\lambda_{t}}\left|y-q_{t}\right|^{2}\right)^{2}},
$$

where $q_{t}$ is chosen such that $\left|q_{t}-p_{t}\right| \ll 1$ and

$$
\left.\nabla_{y} I_{t}(y)\right|_{y=p_{t}}=-\left.t \rho_{t} \nabla_{x} R\left(x, t p_{t}\right)\right|_{x=t p_{t}}-\left.t \nabla_{x} w(x)\right|_{x=t p_{t}}
$$

By direct computation, we have 


$$
\left|q_{t}-p_{t}\right|=O\left(t e^{-\lambda_{t}}\right)
$$

For $y \in B_{2 r_{0}}\left(p_{t}\right)$, we set

$$
\eta_{t}(y)=v_{t}(y)-I_{t}(y)-\left(G_{*, t}(t y)-G_{*, t}\left(t p_{t}\right)\right)
$$

where

$$
G_{*, t}(x)=\rho_{t} R\left(x, t p_{t}\right)+w(x) .
$$

It is easy to see that

$$
\eta_{t}\left(p_{t}\right)=v_{t}\left(p_{t}\right)-I_{t}\left(p_{t}\right)=O\left(t^{2} e^{-\lambda_{t}}\right), \nabla \eta_{t}\left(p_{t}\right)=0
$$

Let

$$
\Lambda_{t,+}=\sqrt{C_{t}} e^{\frac{\lambda_{t}}{2}}, \text { and } \Lambda_{t,-}=\left(\Lambda_{t,+}\right)^{-1}=\frac{e^{-\frac{\lambda_{t}}{2}}}{\sqrt{C_{t}}}
$$

and $\widetilde{\eta}_{t}$ be the scaled function of $\eta_{t}$, that is

$$
\widetilde{\eta}_{t}(z)=\eta_{t}\left(\left(\Lambda_{t,-}\right) z+p_{t}\right) \quad \text { for } \quad|z| \leq 2 R_{0} \Lambda_{t,+}
$$

For $\widetilde{\eta}_{t}(z)$, we have the following estimate

Theorem E. [23, Lemma 7.1] Suppose that the assumptions of Theorem $D$ hold. Then for any $\varepsilon \in(0,1)$, there exists a constant $C_{\varepsilon}>0$, independent of $t>0$ and $z \in B_{2 R_{0} \Lambda_{t,+}}(0)$ such that

$$
\left|\widetilde{\eta}_{t}(z)\right| \leq C_{\varepsilon}\left(t\left\|\widetilde{\phi}_{t}\right\|_{*}+t^{2}\right)(1+|z|)^{\varepsilon}
$$

\section{Uniqueness of the blow up solutions with mass concentration}

To prove Theorem 1.2 is equivalent to prove the local uniqueness of blow up solutions of the equation (2.4). To show it, we argue by contradiction and suppose that (2.4) has two different blow up solutions $u_{t}^{(1)}$ and $u_{t}^{(2)}$, which satisfy $u_{t}^{(1)}, u_{t}^{(2)} \rightarrow w$ in $C_{\text {loc }}(M \backslash\{0\})$, where $w$ is a non-degenerate solution of (2.7). We will use $p_{t}^{(i)}, \lambda_{t}^{(i)}, \bar{u}_{t}^{(i)}, I_{t}^{(i)}, \widetilde{\phi}_{t}^{(i)}, C_{t}^{(i)}$, $q_{t}^{(i)}, v_{t}^{(i)}, \rho_{t}^{(i)}, \eta_{t}^{(i)}, \widetilde{\eta}_{t}^{(i)}, G_{*, t}^{(i)}, \Lambda_{t,+}^{(i)}, \Lambda_{t,-}^{(i)}$ to denote $p_{t}, \lambda_{t}, \bar{u}_{t}, I_{t}, \widetilde{\phi}_{t}, C_{t}, q_{t}, v_{t}, \rho_{t}, \eta_{t}, \widetilde{\eta}_{t}$, $G_{*, t}, \Lambda_{t,+}, \Lambda_{t,-}$ in section 2 corresponding to $u_{t}^{(i)}, i=1,2$, respectively.

From Theorem D, we have $\left|p_{t}^{(i)}\right|=O(t)$ for $i=1,2$. In the following lemma, we shall improve the estimation for $\left|p_{t}^{(1)}-p_{t}^{(2)}\right|$.

Lemma 3.1. $\left|p_{t}^{(1)}-p_{t}^{(2)}\right|=O\left(t^{2} \ln t\right)$. 
Proof. Recall that $v_{t}^{(i)}(y)=u_{t}^{(i)}(t y)-\ln \int_{M} h e^{u_{t}^{(i)}-G_{t}} d v_{g}-\bar{\varphi}(t y)+6 \ln t$ satisfies

$$
\Delta v_{t}^{(i)}+h_{t}(y) e^{v_{t}^{(i)}(y)}=0
$$

where $h_{t}(y)=\rho h(t y)|y-\underline{e}|^{2}|y+\underline{e}|^{2} e^{-R_{t}(t y)+\psi(t y)}$.

On $\partial B_{2 R_{0}}\left(p_{t}^{(i)}\right)$, we have

$$
\begin{aligned}
v_{t}^{(i)}(y)= & -\frac{\rho_{t}^{(i)}}{2 \pi} \ln \left|y-p_{t}^{(i)}\right|+\left(6-\frac{\rho_{t}^{(i)}}{2 \pi}\right) \ln t+G_{*, t}^{(i)}(t y) \\
& -\ln \int_{M} h e^{u_{t}^{(i)}-G_{t}} d v_{g}+\widetilde{\phi}_{t}^{(i)}(t y)-\bar{\varphi}(t y),
\end{aligned}
$$

where $G_{*, t}^{(i)}(x)=\rho_{t}^{(i)} R\left(x, t p_{t}^{(i)}\right)+w(x)$.

For any unit vector $\xi \in \mathbb{R}^{2}$, we apply the Pohozaev identity to (3.1) by multiplying $\xi \cdot \nabla v_{t}^{(i)}$, and obtain

$$
\begin{aligned}
& \sum_{i=1}^{2}(-1)^{i+1} \int_{B_{2 R_{0}\left(p_{t}^{(i)}\right)}}\left(\xi \cdot \nabla h_{t}\right) e^{v_{t}^{(i)}(y)} \\
& =\sum_{i=1}^{2}(-1)^{i+1} \int_{\partial B_{2 R_{0}}\left(p_{t}^{(i)}\right)}\left\{\left(\nu \cdot \nabla v_{t}^{(i)}\right)\left(\xi \cdot \nabla v_{t}^{(i)}\right)-\frac{1}{2}(\nu \cdot \xi)\left|\nabla v_{t}^{(i)}\right|^{2}\right\} \\
& +\sum_{i=1}^{2}(-1)^{i+1} \int_{\partial B_{2 R_{0}}\left(p_{t}^{(i)}\right)}(\nu \cdot \xi) h_{t} e^{v_{t}^{(i)}},
\end{aligned}
$$

where $\nu$ denotes the unit normal of $\partial B_{2 R_{0}}\left(p_{t}^{(i)}\right)$. From (2.12), we have

$$
\left|\nabla_{y} \bar{\varphi}(t y)\right|=t\left|\nabla_{t y} \bar{\varphi}(t y)\right|=O\left(t^{2}|y|\right) \text { for }|y| \leq \frac{r_{0}}{t} .
$$

For the right hand side of (3.3), we can use (3.2), Theorem D, and Theorem E to get

$$
\begin{aligned}
& \text { (RHS) of (3.3) } \\
& =\sum_{i=1}^{2}(-1)^{i+1} \int_{\partial B_{2 R_{0}}\left(p_{t}^{(i)}\right)}\left[\left(\nu \cdot \nabla v_{t}^{(i)}\right)\left(\xi \cdot \nabla v_{t}^{(i)}\right)-\frac{1}{2}(\nu \cdot \xi)\left|\nabla v_{t}^{(i)}\right|^{2} d y+O\left(\sum_{i=1}^{2} e^{-\lambda_{t}^{(i)}}\right)\right] \\
& =\sum_{i=1}^{2}(-1)^{i}\left[\left.t \rho_{t}^{(i)} \xi \cdot \nabla_{x} G_{*, t}^{(i)}(x)\right|_{x=t p_{t}^{(i)}}+O\left(t\left\|\widetilde{\phi}_{t}^{(i)}\right\|_{*}+t^{2}\right)\right]=O\left(t^{2} \ln t\right)
\end{aligned}
$$

For the left hand side of (3.3), by using Theorem D, we get that 


$$
\begin{aligned}
(L H S) & =\sum_{i=1}^{2}(-1)^{i+1} \int_{B_{2 R_{0}}\left(p_{t}^{(i)}\right)}\left(\xi \cdot \frac{\nabla h_{t}\left(p_{t}^{(i)}\right)}{h_{t}\left(p_{t}^{(i)}\right)}\right) h_{t}(y) e^{v_{t}^{(i)}(y)} d y \\
& +\sum_{i=1}^{2}(-1)^{i+1} \int_{B_{2 R_{0}}\left(p_{t}^{(i)}\right)} \xi \cdot\left(\frac{\nabla h_{t}(y)}{h_{t}(y)}-\frac{\nabla h_{t}\left(p_{t}^{(i)}\right)}{h_{t}\left(p_{t}^{(i)}\right)}\right) h_{t}(y) e^{v_{t}^{(i)}(y)} d y \\
& =\sum_{i=1}^{2}(-1)^{i+1} \rho_{t}^{(1)}\left(\xi \cdot \frac{\nabla h_{t}\left(p_{t}^{(i)}\right)}{h_{t}\left(p_{t}^{(i)}\right)}\right) \\
& \left.+\sum_{i=1}^{2}(-1)^{i+1} \int_{B_{2} R_{0}\left(p_{t}^{(i)}\right)} \xi \frac{\nabla h_{t}(y)}{h_{t}(y)}-\frac{\nabla h_{t}\left(p_{t}^{(i)}\right)}{h_{t}\left(p_{t}^{(i)}\right)}\right) \\
& \times \frac{h_{t}(y) e^{\lambda_{t}^{(i)}+\eta_{t}^{(i)}+G_{*, t}^{(i)}(t y)-G_{*, t}^{(i)}\left(t p_{t}^{(i)}\right)}}{\left(1+C_{t}^{(i)} e^{\lambda_{t}^{(i)}}\left|y-q_{t}^{(i)}\right|^{2}\right)^{2}} d y+O\left(t^{2} \ln t\right) .
\end{aligned}
$$

By the change of variable $z=\Lambda_{t,+}^{(i)}\left(y-p_{t}^{(i)}\right)$ and $\int_{B_{2 R_{0} \Lambda_{t,+}^{(i)}}(0) \frac{z_{k}}{\left(1+|z|^{2}\right)^{2}}} d z=0$ for $k=1,2$, we see that

$$
\begin{aligned}
& \int_{B_{2 R_{0}}\left(p_{t}^{(i)}\right)} \xi \cdot\left(\frac{\nabla h_{t}(y)}{h_{t}(y)}-\frac{\nabla h_{t}\left(p_{t}^{(i)}\right)}{h_{t}\left(p_{t}^{(i)}\right)}\right) \frac{h_{t}(y) e^{\lambda_{t}^{(i)}+\eta_{t}^{(i)}+G_{*, t}^{(i)}(t y)-G_{*, t}^{(i)}\left(t p_{t}^{(i)}\right)}}{\left(1+C_{t}^{(i)} e^{\lambda_{t}^{(i)}}\left|y-q_{t}^{(i)}\right|^{2}\right)^{2}} d y \\
& =\int_{B_{2 R_{0}}\left(p_{t}^{(i)}\right)} \xi \cdot\left(\nabla\left(\frac{\nabla h_{t}\left(p_{t}^{(i)}\right)}{h_{t}\left(p_{t}^{(i)}\right)}\right) \cdot\left(y-p_{t}^{(i)}\right)+O\left(\left|y-p_{t}^{(i)}\right|^{2}\right)\right) \\
& \times \frac{h_{t}(y) e^{\lambda_{t}^{(i)}+\eta_{t}^{(i)}+G_{*, t}^{(i)}(t y)-G_{*, t}^{(i)}\left(t p_{t}^{(i)}\right)}}{\left(1+C_{t}^{(i)} e^{\lambda_{t}^{(i)}}\left|y-q_{t}^{(i)}\right|^{2}\right)^{2}} d y \\
& =\int_{B_{2 R_{0} \Lambda_{t,+}^{(i)}(0)}} \xi \cdot\left(\nabla\left(\frac{\nabla h_{t}\left(p_{t}^{(i)}\right)}{h_{t}\left(p_{t}^{(i)}\right)}\right) \cdot\left(\Lambda_{t,-}^{(i)}\right) z+O\left(t^{2}|z|^{2}\right)\right) \\
& \times \frac{h_{t}\left(\left(\Lambda_{t,-}^{(i)}\right) z+p_{t}^{(i)}\right) e^{\widetilde{\eta}_{t}^{(i)}+G_{*, t}^{(i)}\left(t\left(\Lambda_{t,-}^{(i)}\right) z+t p_{t}^{(i)}\right)-G_{*, t}^{(i)}\left(t p_{t}^{(i)}\right)}}{C_{t}^{(i)}\left(1+\left|z+\Lambda_{t,+}^{(i)}\left(p_{t}^{(i)}-q_{t}^{(i)}\right)\right|^{2}\right)^{2}} d z \\
& =\int_{B_{2 R_{0} \Lambda_{t,+}^{(i)}}(0)} \xi \cdot\left(\nabla\left(\frac{\nabla h_{t}\left(p_{t}^{(i)}\right)}{h_{t}\left(p_{t}^{(i)}\right)}\right) \cdot\left(\Lambda_{t,-}^{(i)}\right) z+O\left(t^{2}|z|^{2}\right)\right) \\
& \times \frac{h_{t}\left(p_{t}^{(i)}\right)\left(1+O(t|z|)+O\left(\left|\widetilde{\eta}_{t}^{(i)}\right|\right)+O\left(t^{2}\right)\right)}{C_{t}^{(i)}\left(1+|z|^{2}\right)^{2}} d z
\end{aligned}
$$




$$
=O\left(t^{2} \ln t\right) \quad \text { for } i=1,2 \text {, }
$$

here we used Theorem $\mathrm{E}$ in the last line.

From (3.5)-(3.7), we have

$$
\frac{\nabla h_{t}\left(p_{t}^{(1)}\right)}{h_{t}\left(p_{t}^{(1)}\right)}-\frac{\nabla h_{t}\left(p_{t}^{(2)}\right)}{h_{t}\left(p_{t}^{(2)}\right)}=O\left(t^{2} \ln t\right) .
$$

By using the expression (2.19) of $h_{t}$, we see that

$$
\begin{aligned}
& \frac{\nabla h_{t}\left(p_{t}^{(1)}\right)}{h_{t}\left(p_{t}^{(1)}\right)}-\frac{\nabla h_{t}\left(p_{t}^{(2)}\right)}{h_{t}\left(p_{t}^{(2)}\right)} \\
& =\left.\nabla\left(\ln |y-\underline{e}|^{2}|y+\underline{e}|^{2}\right)\right|_{y=p_{t}^{(1)}}-\left.\nabla\left(\ln |y-\underline{e}|^{2}|y+\underline{e}|^{2}\right)\right|_{y=p_{t}^{(2)}}+O\left(t\left|p_{t}^{(1)}-p_{t}^{(2)}\right|\right) .
\end{aligned}
$$

Note that $\left|p_{t}^{(1)}-p_{t}^{(2)}\right|=O(t)$ from Theorem $\mathrm{D}$, and $\left.\nabla^{2}\left(\ln |y-\underline{e}|^{2}|y+\underline{e}|^{2}\right)\right|_{y=0}$ is invertible. So (3.8) and (3.9) yield that $\left|p_{t}^{(1)}-p_{t}^{(2)}\right|=O\left(t^{2} \ln t\right)$, and thus we complete the proof of Lemma 3.1.

Now we are going to estimate $\left\|u_{t}^{(1)}-u_{t}^{(2)}\right\|_{L^{\infty}(M)}$.

\section{Lemma 3.2.}

$$
\left\|u_{t}^{(1)}-u_{t}^{(2)}\right\|_{L^{\infty}(M)}=O(t \ln t)
$$

Proof. We note that $M \backslash B_{2 R_{0} t}\left(t p_{t}^{(2)}\right) \subseteq M \backslash B_{2 R_{1} t}\left(t p_{t}^{(1)}\right)$ for some $R_{1}>0$. For $x \in$ $M \backslash B_{2 R_{0} t}\left(t p_{t}^{(1)}\right)$, we see from Theorem $\mathrm{D}$ that

$$
\begin{aligned}
& u_{t}^{(1)}(x)-u_{t}^{(2)}(x) \\
& =\left(w(x)+\rho_{t}^{(1)} G\left(x, t p_{t}^{(1)}\right)+\widetilde{\phi}_{t}^{(1)}(x)\right)-\left(w(x)+\rho_{t}^{(2)} G\left(x, t p_{t}^{(2)}\right)+\widetilde{\phi}_{t}^{(2)}(x)\right) \\
& =\rho_{t}^{(1)} G\left(x, t p_{t}^{(1)}\right)-\rho_{t}^{(2)} G\left(x, t p_{t}^{(2)}\right)+O(t \ln t) .
\end{aligned}
$$

Together with Theorem D and Theorem E, we have for some $\theta \in(0,1)$,

$$
\begin{aligned}
u_{t}^{(1)}(x)-u_{t}^{(2)}(x) & =-\frac{\rho_{t}^{(1)}}{2 \pi}\left(\ln \left|x-t p_{t}^{(1)}\right|-\ln \left|x-t p_{t}^{(2)}\right|\right)+O(t \ln t) \\
& =\frac{O\left(t\left|p_{t}^{(1)}-p_{t}^{(2)}\right|\right)}{\theta\left|x-t p_{t}^{(1)}\right|+(1-\theta)\left|x-t p_{t}^{(2)}\right|}+O(t \ln t) \\
& =O\left(\left|p_{t}^{(1)}-p_{t}^{(2)}\right|\right)+O(t \ln t)=O(t \ln t) \text { for } x \in M \backslash B_{2 R_{0} t}\left(t p_{t}^{(1)}\right) .
\end{aligned}
$$

We note that $B_{2 R_{0} t}\left(t p_{t}^{(2)}\right) \subseteq B_{2 R_{2} t}\left(t p_{t}^{(1)}\right)$ for some $R_{2}>0$. For $y \in B_{2 R_{0}}\left(p_{t}^{(1)}\right)$, we see that 


$$
\begin{aligned}
\eta_{t}^{(1)}(y)-\eta_{t}^{(2)}(y) & \\
= & \left(v_{t}^{(1)}(y)-I_{t}^{(1)}(y)-\left(G_{*, t}^{(1)}(t y)-G_{*, t}^{(1)}\left(t p_{t}^{(1)}\right)\right)\right) \\
& \quad-\left(v_{t}^{(2)}(y)-I_{t}^{(2)}(y)-\left(G_{*, t}^{(2)}(t y)-G_{*, t}^{(2)}\left(t p_{t}^{(2)}\right)\right)\right) \\
= & u_{t}^{(1)}(t y)-\ln \int_{M} h e^{u_{t}^{(1)}-G_{t}} d v_{g}-\lambda_{t}^{(1)}+2 \ln \left(1+C_{t}^{(1)} e^{\lambda_{t}^{(1)}}\left|y-q_{t}^{(1)}\right|^{2}\right) \\
& -\left(u_{t}^{(2)}(t y)-\ln \int_{M} h e^{u_{t}^{(2)}-G_{t}} d v_{g}-\lambda_{t}^{(2)}+2 \ln \left(1+C_{t}^{(2)} e^{\lambda_{t}^{(2)}}\left|y-q_{t}^{(2)}\right|^{2}\right)\right) \\
& +O(t) .
\end{aligned}
$$

By Theorem D, we have

$$
\begin{gathered}
\int_{M} h e^{u_{t}^{(1)}-G_{t}} d v_{g}-\int_{M} h e^{u_{t}^{(2)}-G_{t}} d v_{g}=O(t), \\
\lambda_{t}^{(i)}+2 \ln t+2 \ln C_{t}^{(i)}+8 \pi R\left(t p_{t}^{(i)}, t p_{t}^{(i)}\right) \\
-\ln \left(\frac{\rho}{\rho-8 \pi} \int_{M} h e^{w}\right)+w\left(t p_{t}^{(i)}\right)=O(t \ln t),
\end{gathered}
$$

and

$$
\begin{aligned}
C_{t}^{(1)}-C_{t}^{(2)} & =\frac{\rho h\left(t p_{t}^{(1)}\right)\left|p_{t}^{(1)}-\underline{e}\right|^{2}\left|p_{t}^{(1)}+\underline{e}\right|^{2} e^{-R_{t}\left(t p_{t}^{(1)}\right)+\psi\left(t p_{t}^{(1)}\right)}}{8} \\
& -\frac{\rho h\left(t p_{t}^{(2)}\right)\left|p_{t}^{(2)}-\underline{e}\right|^{2}\left|p_{t}^{(2)}+\underline{e}\right|^{2} e^{-R_{t}\left(t p_{t}^{(2)}\right)+\psi\left(t p_{t}^{(2)}\right)}}{8} \\
& =O\left(\left|p_{t}^{(1)}-p_{t}^{(2)}\right|\right)=O\left(t^{2} \ln t\right),
\end{aligned}
$$

which imply

$$
\lambda_{t}^{(1)}-\lambda_{t}^{(2)}=O(t \ln t)
$$

For $y \in B_{2 R_{0}}\left(p_{t}^{(1)}\right)$, we want to estimate

$$
2 \ln \left(1+C_{t}^{(1)} e^{\lambda_{t}^{(1)}}\left|y-q_{t}^{(1)}\right|^{2}\right)-2 \ln \left(1+C_{t}^{(2)} e^{\lambda_{t}^{(2)}}\left|y-q_{t}^{(2)}\right|^{2}\right) .
$$

In view of (2.25) and Lemma 3.1, we have

$$
\left|q_{t}^{(1)}-q_{t}^{(2)}\right| \leq \sum_{i=1}^{2}\left|p_{t}^{(i)}-q_{t}^{(i)}\right|+\left|p_{t}^{(1)}-p_{t}^{(2)}\right|=O\left(t^{2} \ln t\right)
$$

Let $y=q_{t}^{(1)}+\Lambda_{t,-}^{(1)} z$. Then we have for $y \in B_{2 R_{0}}\left(p_{t}^{(1)}\right)$, 


$$
\begin{aligned}
& 2 \ln \left(1+C_{t}^{(1)} e^{\lambda_{t}^{(1)}}\left|y-q_{t}^{(1)}\right|^{2}\right)-2 \ln \left(1+C_{t}^{(2)} e^{\lambda_{t}^{(2)}}\left|y-q_{t}^{(2)}\right|^{2}\right) \\
& =2 \ln \left(1+|z|^{2}\right)-2 \ln \left(1+\frac{C_{t}^{(2)} e^{\lambda_{t}^{(2)}}}{C_{t}^{(1)} e^{\lambda_{t}^{(1)}}}\left|\Lambda_{t,+}^{(1)}\left(y-q_{t}^{(1)}\right)+\Lambda_{t,+}^{(1)}\left(q_{t}^{(1)}-q_{t}^{(2)}\right)\right|^{2}\right) \\
& =2 \ln \left(1+|z|^{2}\right)-2 \ln \left(1+(1+O(t \ln t))|z+O(t \ln t)|^{2}\right)=O(t \ln t) .
\end{aligned}
$$

By Theorem E, we have

$$
\eta_{t}^{(1)}(y)-\eta_{t}^{(2)}(y)=O(t \ln t) \text { for } y \in B_{2 R_{0}}\left(p_{t}^{(1)}\right)
$$

From (3.12)-(3.19), we have

$$
u_{t}^{(1)}(x)-u_{t}^{(2)}(x)=O(t \ln t) \quad \text { for } \quad x \in B_{2 R_{0} t}\left(t p_{t}^{(1)}\right) .
$$

By (3.11) and (3.20), we complete the proof of Lemma 3.2.

Let

$$
\zeta_{t}(x)=\frac{u_{t}^{(1)}(x)-u_{t}^{(2)}(x)}{\left\|u_{t}^{(1)}-u_{t}^{(2)}\right\|_{L^{\infty}(M)}}
$$

and

$$
\widetilde{\zeta}_{t}(z)=\zeta_{t}\left(t \Lambda_{t,-}^{(1)} z+t p_{t}^{(1)}\right)-\frac{\int_{M} h e^{u_{t}^{(1)}-G_{t}} \zeta_{t} d v_{g}}{\int_{M} h e^{u_{t}^{(1)}-G_{t}} d v_{g}}
$$

Now we have the following estimation for the scaled function $\widetilde{\zeta}_{t}$.

Lemma 3.3. There are constants $b_{0}, b_{1}$, and $b_{2}$ satisfying

$$
\widetilde{\zeta}_{t}(z) \rightarrow \widetilde{\zeta}_{0}(z)=b_{0} Y_{0}(z)+b_{1} Y_{1}(z)+b_{2} Y_{2}(z) \quad \text { in } \quad C_{l o c}^{0}\left(\mathbb{R}^{2}\right)
$$

where $Y_{0}(z)=\frac{1-|z|^{2}}{1+|z|^{2}}, Y_{1}(z)=\frac{z_{1}}{1+|z|^{2}}, Y_{2}(z)=\frac{z_{2}}{1+|z|^{2}}$.

Proof. First, we see that

$$
\begin{aligned}
0 & =\Delta_{M} \zeta_{t}+\frac{1}{\left\|u_{t}^{(1)}-u_{t}^{(2)}\right\|_{L^{\infty}(M)}}\left(\frac{\rho h(x) e^{u_{t}^{(1)}(x)-G_{t}(x)}}{\int_{M} h e^{u_{t}^{(1)}-G_{t}} d v_{g}}-\frac{\rho h(x) e^{u_{t}^{(2)}(x)-G_{t}(x)}}{\int_{M} h e^{u_{t}^{(2)}-G_{t}} d v_{g}}\right) \\
& =\Delta_{M} \zeta_{t}+\frac{\rho h(x) e^{u_{t}^{(1)}(x)-G_{t}(x)}}{\left\|u_{t}^{(1)}-u_{t}^{(2)}\right\|_{L^{\infty}(M)} \int_{M} h e^{u_{t}^{(1)}-G_{t}} d v_{g}}\left(1-\frac{e^{u_{t}^{(2)}(x)-u_{t}^{(1)}(x)} \int_{M} h e^{u_{t}^{(1)}-G_{t}}}{\int_{M} h e^{u_{t}^{(2)}-G_{t}} d v_{g}}\right) \\
& =\Delta_{M} \zeta_{t}+\frac{\rho h(x) e^{u_{t}^{(1)}(x)-G_{t}(x)}}{\left\|u_{t}^{(1)}-u_{t}^{(2)}\right\|_{L^{\infty}(M)} \int_{M} h e^{u_{t}^{(1)}-G_{t}} d v_{g}}(1
\end{aligned}
$$




$$
\begin{aligned}
& \left.=\frac{\left(1+u_{t}^{(2)}(x)-u_{t}^{(1)}(x)+O\left(\left\|u_{t}^{(1)}-u_{t}^{(2)}\right\|_{L^{\infty}(M)}^{2}\right)\right) \int_{M} h e^{u_{t}^{(1)}-G_{t}} d v_{g}}{\int_{M} h e^{u_{t}^{(1)}}-G_{t}\left(1+u_{t}^{(2)}(x)-u_{t}^{(1)}(x)+O\left(\left\|u_{t}^{(1)}-u_{t}^{(2)}\right\|_{L^{\infty}(M)}^{2}\right)\right) d v_{g}}\right) \\
= & \Delta_{M} \zeta_{t}+\frac{\rho h(x) e^{u_{t}^{(1)}(x)-G_{t}(x)}}{\int_{M} h e^{u_{t}^{(1)}-G_{t}} d v_{g}}\left(\zeta_{t}-\frac{\int_{M} h e^{u_{t}^{(1)}-G_{t}} \zeta_{t} d v_{g}}{\int_{M} h e^{u_{t}^{(1)}-G_{t}} d v_{g}}+O\left(\left\|u_{t}^{(1)}-u_{t}^{(2)}\right\|_{L^{\infty}(M)}\right)\right) .
\end{aligned}
$$

By using the change of variables $y=t \Lambda_{t,-}^{(1)} z+t p_{t}^{(1)},(2.17),(2.26)$, we have

$$
\begin{aligned}
& \Delta_{z} \widetilde{\zeta}_{t}(z)=-\frac{t^{6}\left(\Lambda_{t,-}^{(1)}\right)^{2} \rho h\left(t \Lambda_{t,-}^{(1)} z+t p_{t}^{(1)}\right) e^{u_{t}^{(1)}\left(t \Lambda_{t,-}^{(1)} z+t p_{t}^{(1)}\right)-R_{t}\left(t \Lambda_{t,-}^{(1)} z+t p_{t}^{(1)}\right)}}{\int_{M} h e^{u_{t}^{(1)}-G_{t}} d v_{g}} \\
& \times\left|\Lambda_{t,-}^{(1)} z+p_{t}^{(1)}-\underline{e}\right|^{2}\left|\Lambda_{t,-}^{(1)} z+p_{t}^{(1)}+\underline{e}\right|^{2}\left(\widetilde{\zeta}_{t}(z)+O\left(\left\|u_{t}^{(1)}-u_{t}^{(2)}\right\|_{L^{\infty}(M)}\right)\right) \\
& =-\left(\Lambda_{t,-}^{(1)}\right)^{2} h_{1}\left(t \Lambda_{t,-}^{(1)} z+t p_{t}^{(1)}\right) e^{v_{t}^{(1)}\left(\Lambda_{t,-}^{(1)} z+p_{t}^{(1)}\right)} \\
& \times\left|\Lambda_{t,-}^{(1)} z+p_{t}^{(1)}-\underline{e}\right|^{2}\left|\Lambda_{t,-}^{(1)} z+p_{t}^{(1)}+\underline{e}\right|^{2}\left(\widetilde{\zeta}_{t}(z)+O\left(\left\|u_{t}^{(1)}-u_{t}^{(2)}\right\|_{L^{\infty}(M)}\right)\right) \\
& =-\left(\frac{1}{C_{t}^{(1)}}\right) \frac{h_{1}\left(t \Lambda_{t,-}^{(1)} z+t p_{t}^{(1)}\right) e^{G_{*, t}^{(1)}\left(t \Lambda_{t,-}^{(1)} z+t p_{t}^{(1)}\right)-G_{*, t}^{(1)}\left(t p_{t}^{(1)}\right)+\widetilde{\eta}_{t}^{(1)}(z)}}{\left(1+\left|z+\Lambda_{t,+}^{(1)}\left(p_{t}^{(1)}-q_{t}^{(1)}\right)\right|^{2}\right)^{2}} \\
& \times\left|\Lambda_{t,-}^{(1)} z+p_{t}^{(1)}-\underline{e}\right|^{2}\left|\Lambda_{t,-}^{(1)} z+p_{t}^{(1)}+\underline{e}\right|^{2}\left(\widetilde{\zeta}_{t}(z)+O\left(\left\|u_{t}^{(1)}-u_{t}^{(2)}\right\|_{L^{\infty}(M)}\right)\right) \\
& =\frac{-8 h_{1}\left(t \Lambda_{t,-}^{(1)} z+t p_{t}^{(1)}\right)}{h_{1}\left(t p_{t}^{(1)}\right)} \frac{\left|\Lambda_{t,-}^{(1)} z+p_{t}^{(1)}-\underline{e}\right|^{2}\left|\Lambda_{t,-}^{(1)} z+p_{t}^{(1)}+\underline{e}\right|^{2}}{\left|p_{t}^{(1)}-\underline{e}\right|^{2}\left|p_{t}^{(1)}+\underline{e}\right|^{2}} \\
& \times \frac{\left(\widetilde{\zeta}_{t}(z)+O\left(\left\|u_{t}^{(1)}-u_{t}^{(2)}\right\|_{L^{\infty}(M)}\right)\right)\left(1+O\left(\left|\widetilde{\eta}_{t}(z)\right|\right)+O\left(t^{2}|z|\right)\right)}{\left(1+\left|z+\Lambda_{t,+}^{(1)}\left(p_{t}^{(1)}-q_{t}^{(1)}\right)\right|^{2}\right)^{2}} .
\end{aligned}
$$

Together with (2.25), Lemma 3.2, and Theorem E, we have for $z \in B_{2 \Lambda_{t,+} R_{0}}(0)$,

$$
\Delta_{z} \widetilde{\zeta}_{t}(z)+\frac{8 \widetilde{\zeta}_{t}(z)}{\left(1+|z|^{2}\right)^{2}}=-\frac{8 \widetilde{\zeta}_{t} \nabla \ln H_{t}\left(p_{t}^{(1)}\right) \cdot\left(\Lambda_{t,-}^{(1)} z\right)+O(t \ln t)+O\left(t^{2}|z|^{2}\right)}{\left(1+|z|^{2}\right)^{2}}
$$

where

$$
H_{t}(x)=h_{1}(t x)|x-\underline{e}|^{2}|x+\underline{e}|^{2} .
$$

Since $\widetilde{\zeta}_{t}$ is uniformly bounded, there is a function $\widetilde{\zeta}_{0}$ such that $\widetilde{\zeta}_{t} \rightarrow \widetilde{\zeta}_{0}$ in $C_{\text {loc }}\left(\mathbb{R}^{2}\right)$, where

$$
\left\{\begin{array}{l}
\Delta \widetilde{\zeta}_{0}+\frac{8 \widetilde{\zeta}_{0}}{\left(1+|z|^{2}\right)^{2}}=0 \text { in } \mathbb{R}^{2} \\
\left\|\widetilde{\zeta}_{0}\right\|_{L^{\infty}\left(\mathbb{R}^{2}\right)} \leq c \text { for some constant } c>0
\end{array}\right.
$$


By [1, Proposition 1], we see that $\widetilde{\zeta}_{0}(z)=\sum_{i=0}^{2} b_{i} Y_{i}(z)$ for some constants $b_{i} \in \mathbb{R}^{2}$, $i=0,1,2$. This completes the proof of Lemma 3.3.

In the following lemma, we observe the behavior of $\zeta_{t}$ in $M \backslash\{0\}$.

Lemma 3.4. (i) $\zeta_{t} \rightarrow 0$ in $C_{l o c}^{0}(M \backslash\{0\})$,

(ii) $\lim _{t \rightarrow 0}\left(\int_{M} h e^{u_{t}^{(1)}-G_{t}} \zeta_{t} d v_{g}\right)=0$.

Proof. We recall from (3.23) that in $M$,

$$
\Delta_{M} \zeta_{t}+\frac{\rho h(x) e^{u_{t}^{(1)}-G_{t}}}{\int_{M} h e^{u_{t}^{(1)}-G_{t}} d v_{g}}\left(\zeta_{t}-\frac{\int_{M} h e^{u_{t}^{(1)}-G_{t}} \zeta_{t} d v_{g}}{\int_{M} h e^{u_{t}^{(1)}-G_{t}} d v_{g}}+O\left(\left\|u_{t}^{(1)}-u_{t}^{(2)}\right\|_{L^{\infty}(M)}\right)=0 .\right.
$$

Since $\left\|\zeta_{t}\right\|_{L^{\infty}(M)} \leq 1$, we see that there is a function $\zeta_{*}$ satisfying

$$
\zeta_{t} \rightarrow \zeta_{*} \text { in } C_{\mathrm{loc}}(M \backslash\{0\})
$$

From Theorem D, we have

$$
\lim _{t \rightarrow 0} \int_{M} h e^{u_{t}^{(1)}-G_{t}} d v_{g}=\frac{\rho}{\rho-8 \pi} \int_{M} h e^{w} d v_{g}
$$

For any small fixed $r \in(0,1)$, we see from Theorem $\mathrm{D}$ that

$$
\begin{aligned}
\int_{M} h e^{u_{t}^{(1)}-G_{t}} \zeta_{t} d v_{g}= & {\left[\int_{M \backslash B_{r}(0)}+\int_{B_{r}(0) \backslash B_{2 R_{0}}\left(t p_{t}^{(1)}\right)}\right] h e^{u_{t}^{(1)}-G_{t}} \zeta_{t} d v_{g} } \\
& +\int_{B_{2 R_{0} t}\left(t p_{t}^{(1)}\right)} h e^{u_{t}^{(1)}-G_{t}}\left(\zeta_{t}-\frac{\int_{M} h e^{u_{t}^{(1)}-G_{t}} \zeta_{t} d v_{g}}{\int_{M} h e^{u_{t}^{(1)}-G_{t}} d v_{g}}\right) d v_{g} \\
& +\int_{B_{2 R_{0} t}\left(t p_{t}^{(1)}\right)} h e^{u_{t}^{(1)}-G_{t}} d v_{g} \frac{\int_{M} h e^{u_{t}^{(1)}-G_{t}} \zeta_{t} d v_{g}}{\int_{M} h e^{u_{t}^{(1)}-G_{t}} d v_{g}} \\
& =\int_{M} h e^{w} \zeta_{*} d v_{g}+\frac{1}{\rho}\left(\int_{M} h e^{u_{t}^{(1)}-G_{t}} d v_{g}\right)_{B_{2} R_{0} t} \int_{\left(t p_{t}^{(1)}\right)}\left(-\Delta_{M} \zeta_{t}\right) d v_{g} \\
& +\frac{8 \pi}{\rho} \int_{M} h e^{u_{t}^{(1)}-G_{t}} \zeta_{t} d v_{g}+o(1)+O\left(r^{2}\right)+O\left(\left\|u_{t}^{(1)}-u_{t}^{(2)}\right\|_{L^{\infty}(M)}\right) .
\end{aligned}
$$

By using the change of variable $x=t\left(\Lambda_{t,-}^{(1)} z+p_{t}^{(1)}\right)$, we note that as $t \rightarrow 0$, 


$$
\begin{aligned}
\int_{B_{2 R_{0} t}\left(t p_{t}^{(1)}\right)}-\Delta_{x} \zeta_{t}(x) d x & =\int_{B_{2 R_{0} \Lambda_{t,+}^{(1)}}(0)}-\Delta_{z} \widetilde{\zeta}_{t}(z) d z \\
= & \int_{B_{2 R_{0} \Lambda_{t,+}^{(1)}}(0)} \frac{8 \widetilde{\zeta}_{t}(z)+O(t|z|)+o(1)}{\left(1+|z|^{2}\right)^{2}} d z=o(1),
\end{aligned}
$$

since $\widetilde{\zeta}_{t} \rightarrow \sum_{j=0}^{2} b_{j} Y_{j}$ in $C_{\text {loc }}\left(\mathbb{R}^{2}\right)$ and $\int_{\mathbb{R}^{2}} \frac{Y_{i}}{\left(1+|z|^{2}\right)^{2}}=0$ for $i=0,1,2$. So we obtain from (3.30) and (3.31) that

$$
\left(1-\frac{8 \pi}{\rho}\right) \int_{M} h e^{u_{t}^{(1)}-G_{t}} \zeta_{t} d v_{g}=\int_{M} h e^{w} \zeta_{*} d v_{g}+o(1),
$$

which implies

$$
\int_{M} h e^{u_{t}^{(1)}-G_{t}} \zeta_{t} d v_{g}=\left(\frac{\rho}{\rho-8 \pi}\right) \int_{M} h e^{w} \zeta_{*} d v_{g}+o(1) .
$$

Then we have

$$
\Delta_{M} \zeta_{*}+\frac{(\rho-8 \pi) h e^{w}}{\int_{M} h e^{w} d v_{g}}\left(\zeta_{*}-\frac{\int_{M} h e^{w} \zeta_{*} d v_{g}}{\int_{M} h e^{w} d v_{g}}\right)=0 \text { in } \quad M \backslash\{0\}
$$

Since $\left\|\zeta_{*}\right\|_{L^{\infty}(M)} \leq 1$, the above equation (3.33) holds in $M$. Moreover, we note that

$$
\int_{M} \zeta_{t} d v_{g}=\frac{\int_{M}\left(u_{t}^{(1)}-u_{t}^{(2)}\right) d v_{g}}{\left\|u_{t}^{(1)}-u_{t}^{(2)}\right\|_{L^{\infty}(M)}}=0
$$

and thus $\int_{M} \zeta_{*} d v_{g}=0$. Together with non-degeneracy condition for $w$, we obtain $\zeta_{*} \equiv 0$. In view of (3.28) and (3.32), we complete the proof of Lemma 3.4.

To connect the behavior of $\zeta_{t}$ in $M \backslash\{0\}$ and in a small neighborhood of 0 , we need the following result.

Lemma 3.5. [25] (i) If $\frac{t R_{0}}{2} \leq\left|x_{2}-t p_{t}^{(1)}\right| \leq\left|x_{1}-t p_{t}^{(1)}\right| \leq r_{0}$, then

$$
\begin{aligned}
\zeta_{t}\left(x_{1}\right)-\zeta_{t}\left(x_{2}\right)= & O\left(\ln \frac{\left|x_{1}-t p_{t}^{(1)}\right|}{\left|x_{2}-t p_{t}^{(1)}\right|} \int_{B_{2 R_{0} \Lambda_{t,+}^{(1)}}(0)} \Delta \widetilde{\zeta}_{t} d z\right) \\
& +O\left(\left|x_{1}-t p_{t}^{(1)}\right| \ln \left|x_{1}-t p_{t}^{(1)}\right|\right)+O\left(t^{\frac{\alpha}{2}} \ln t\right)
\end{aligned}
$$


(ii) If $t^{2} R_{0} \leq\left|x-t p_{t}^{(1)}\right| \leq \frac{t R_{0}}{2}$, then

$$
\begin{aligned}
& \zeta_{t}(x)-\zeta_{t}\left(t p_{t}^{(1)}\right)=O\left(\int_{B_{2 R_{0} \Lambda_{t,+}^{(1)}(0)}}(\ln |z|) \Delta \widetilde{\zeta}_{t} d z\right)+O\left(\ln \frac{\left|x-t p_{t}^{(1)}\right|}{t^{2}} \int_{B_{2 R_{0} \Lambda_{t,+}^{(1)}(0)}} \Delta \widetilde{\zeta}_{t} d z\right) \\
& +O\left(\left(\frac{\left|x-t p_{t}^{(1)}\right|}{t^{2}}\right)^{-\frac{\alpha}{2}} \ln \left(\frac{\left|x-t p_{t}^{(1)}\right|}{t^{2}}\right)\right)+O(t \ln t) \text {. }
\end{aligned}
$$

Proof. For any function $g$ satisfying $g(z)(1+|z|)^{1+\frac{\alpha}{2}} \in L^{2}\left(\mathbb{R}^{2}\right)$, we recall the following estimation (see [9]): there is a constant $c>0$, independent of $x \in \mathbb{R}^{2} \backslash B_{2}(0)$ and $g$, such that

$$
\left|\int_{\mathbb{R}^{2}}(\ln |x-z|-\ln |x|) g(z) \mathrm{d} z\right| \leq c|x|^{-\frac{\alpha}{2}}(\ln |x|+1)\left\|g(z)(1+|z|)^{1+\frac{\alpha}{2}}\right\|_{L^{2}\left(\mathbb{R}^{2}\right)} .
$$

Together with the Green representation formula, Lemma 3.5 can be obtained. See [25] for the detail.

Let $\chi_{t}$ be a cut-off function satisfying $0 \leq \chi_{t} \leq 1,\left|\nabla \chi_{t}\right|=O(t),\left|\nabla^{2} \chi_{t}\right|=O\left(t^{2}\right)$, and

$$
\chi_{t}(z)=\chi_{t}(|z|)= \begin{cases}1 & \text { if }|z| \leq R_{0} \Lambda_{t,+}^{(1)} \\ 0 & \text { if }|z| \geq 2 R_{0} \Lambda_{t,+}^{(1)}\end{cases}
$$

Then we have the following result.

\section{Lemma 3.6.}

(i) $\int_{B_{2 R_{0} \Lambda_{t,+}^{(1)}}(0)} \frac{\widetilde{\zeta}_{t}(z) \chi_{t}(z)}{\left(1+|z|^{2}\right)^{2}} d z=O(t \ln t)$,

(ii) $\int_{B_{2 R_{0} \Lambda_{t,+}^{(1)}}(0)} \Delta \widetilde{\zeta}_{t} d z=\int_{B_{2 R_{0} t\left(t p_{t}^{(1)}\right)}} \Delta \zeta_{t} d x=O(t \ln t)$,

(iii) $\lim _{t \rightarrow 0}\left\|\zeta_{t}\right\|_{L^{\infty}\left(M \backslash B_{\frac{t R_{0}}{2}}\left(t p_{t}^{(1)}\right)\right)}=0$,

(iv) $\lim _{t \rightarrow 0} \int_{B_{2 R_{0} \Lambda_{t,+}^{(1)}}(0)} \frac{\widetilde{\zeta}_{t}^{2}(z) Y_{0}(z) \chi_{t}(z)}{\left(1+|z|^{2}\right)^{2}} d z=o(1)$,

(v) $b_{0}=0$.

Proof. (i) We note that $\eta_{1}(z)=-\frac{2}{\left(1+|z|^{2}\right)}$ satisfies

$$
\Delta \eta_{1}+\frac{8 \eta_{1}}{\left(1+|z|^{2}\right)^{2}}=-\frac{8}{\left(1+|z|^{2}\right)^{2}} \text { in } \mathbb{R}^{2}
$$


From (3.25), we recall the following equation:

$$
\Delta_{z} \widetilde{\zeta}_{t}(z)+\frac{8 \widetilde{\zeta}_{t}(z)}{\left(1+|z|^{2}\right)^{2}}=-\frac{8\left(\Lambda_{t,-}^{(1)}\right) \widetilde{\zeta}_{t} \nabla \ln H_{t}\left(p_{t}^{(1)}\right) \cdot z+O(t \ln t)+O\left(t^{2}|z|^{2}\right)}{\left(1+|z|^{2}\right)^{2}} .
$$

Multiplying both sides of (3.25) by $\eta_{1} \chi_{t}$ and using the integration by parts, we have

$$
\begin{aligned}
0= & \int_{B_{2 R_{0} \Lambda_{t,+}^{(1)}}^{(0)}} \widetilde{\zeta}_{t}\left(\Delta\left(\eta_{1} \chi_{t}\right)+\frac{8 \eta_{1} \chi_{t}}{\left(1+|z|^{2}\right)^{2}}\right) d z+O(t \ln t) \\
= & \int_{\substack{B_{2 R_{0} \Lambda_{t,+}^{(1)}}^{(0)}\\
}} \widetilde{\zeta}_{t}\left[\left(\Delta \eta_{1}+\frac{8 \eta_{1}}{\left(1+|z|^{2}\right)^{2}}\right) \chi_{t}+2 \nabla \eta_{1} \cdot \nabla \chi_{t}+\eta_{1} \Delta \chi_{t}\right] d z \\
& +O(t \ln t) .
\end{aligned}
$$

Together with (3.38), we obtain

$$
\int_{B_{2 R_{0} \Lambda_{t,+}^{(1)}}^{(0)}} \frac{8 \widetilde{\zeta}_{t} \chi_{t}}{\left(1+|z|^{2}\right)^{2}} d z=O(t \ln t)
$$

(ii) By integrating (3.25) and using (3.40), we have Lemma 3.6-(ii).

(iii) By Lemma 3.5-(i) and Lemma 3.4-(i), we see that if $\frac{t R_{0}}{2} \leq\left|x-t p_{t}^{(1)}\right| \leq r_{0}$ and $\left|x^{\prime}-t p_{t}^{(1)}\right|=r$, then

$$
\zeta_{t}(x)=\zeta_{t}\left(x^{\prime}\right)+O\left(\ln t \int_{B_{2 R_{0} \Lambda_{t,+}^{(1)}}(0)} \Delta \widetilde{\zeta}_{t} d z\right)+O(r \ln r)+o(1)
$$

for any small $r>0$. Together with Lemma 3.6-(ii), we can get that Lemma 3.6-(iii).

(iv) We note that $\eta_{2}(z)=\frac{4}{3} \ln \left(1+|z|^{2}\right)\left(\frac{1-|z|^{2}}{1+|z|^{2}}\right)+\frac{8}{3\left(1+|z|^{2}\right)}$ satisfies

$$
\Delta \eta_{2}+\frac{8 \eta_{2}}{\left(1+|z|^{2}\right)^{2}}=\frac{16 Y_{0}(z)}{\left(1+|z|^{2}\right)^{2}} \text { in } \mathbb{R}^{2}
$$

Multiplying both sides of (3.25) by $\eta_{2} \chi_{t}$ and using the integration by parts, we have

$$
0=\int_{B_{2 R_{0} \Lambda_{t,+}^{(1)}(0)}} \widetilde{\zeta}_{t}\left[\left(\Delta \eta_{2}+\frac{8 \eta_{2}}{\left(1+|z|^{2}\right)^{2}}\right) \chi_{t}+2 \nabla \eta_{2} \cdot \nabla \chi_{t}+\eta_{2} \Delta \chi_{t}\right] d z+O(t \ln t)
$$

Fix a point $e_{t} \in \partial B_{R_{0} \Lambda_{t,+}^{(1)}}(0)$. Then (3.42) implies 


$$
\begin{aligned}
\int_{B_{2 R_{0} \Lambda_{t,+}^{(1)}(0)}} \frac{16 Y_{0} \widetilde{\zeta}_{t} \chi_{t}}{\left(1+|z|^{2}\right)^{2}} d z & \left.=\int_{B_{2 R_{0} \Lambda_{t,+}^{(1)}(0)}}\left(\widetilde{\zeta}_{t}(z)-\widetilde{\zeta}_{t}\left(e_{t}\right)\right)\right)\left(2 \nabla \eta_{2} \cdot \nabla \chi_{t}+\eta_{2} \Delta \chi_{t}\right) d z \\
& -\widetilde{\zeta}_{t}\left(e_{t}\right) \int_{B_{2 R_{0} \Lambda_{t,+}^{(1)}(0)}}\left(2 \nabla \eta_{2} \cdot \nabla \chi_{t}+\eta_{2} \Delta \chi_{t}\right) d z+O(t \ln t) .
\end{aligned}
$$

Together with Lemma 3.5-(i) and Lemma 3.6-(ii), we have

$$
\begin{aligned}
& \int_{B_{2 R_{0} \Lambda_{t,+}^{(1)}(0)}} \frac{16 Y_{0} \widetilde{\zeta}_{t} \chi_{t}}{\left(1+|z|^{2}\right)^{2}} d z \\
= & \int_{R_{0} \Lambda_{t,+}^{(1)} \leq|z| \leq 2 R_{0} \Lambda_{t,+}^{(1)}} O\left(t^{\frac{\alpha}{2}} \ln t\right)\left(\frac{t}{|z|}+|\ln | z|| t^{2}\right) d z+O\left(\widetilde{\zeta}_{t}\left(e_{t}\right)\right)+O(t \ln t),
\end{aligned}
$$

here we used $\int_{B_{2 R_{0} \Lambda_{t,+}^{(1)}}(0)} \eta_{2} \Delta \chi_{t} d z=-\int_{B_{2 R_{0} \Lambda_{t,+}^{(1)}}(0)} \nabla \eta_{2} \cdot \nabla \chi_{t} d z$.

By applying Lemma 3.4-(ii) and Lemma 3.6-(iii), we obtain $\lim _{t \rightarrow 0} \widetilde{\zeta}_{t}\left(e_{t}\right)=0$, and thus

$$
\int_{B_{2 R_{0} \Lambda_{t,+}^{(1)}}(0)} \frac{16 Y_{0} \widetilde{\zeta}_{t} \chi_{t}}{\left(1+|z|^{2}\right)^{2}} d z=o(1) \text { as } t \rightarrow 0
$$

So we obtain Lemma 3.6-(iv).

(v) By Lemma 3.3 and Lemma 3.6-(iv), we have

$$
b_{0} \equiv 0 \text {. }
$$

So we complete the proof of Lemma 3.6.

Let

$$
\widetilde{u}_{t}^{(i)}=u_{t}^{(i)}-\ln \int_{M} h e^{u_{t}^{(i)}-G_{t}} d v_{g} \text { for } i=1,2
$$

We note that

$$
\begin{aligned}
& \widetilde{u}_{t}^{(1)}-\widetilde{u}_{t}^{(2)} \\
& \quad=u_{t}^{(1)}-u_{t}^{(2)}-\ln \int_{M} h e^{u_{t}^{(1)}-G_{t}} d v_{g}+\ln \int_{M} h e^{u_{t}^{(2)}-G_{t}} d v_{g}
\end{aligned}
$$




$$
\begin{aligned}
= & u_{t}^{(1)}-u_{t}^{(2)}-\ln \int_{M} h e^{u_{t}^{(1)}-G_{t}} d v_{g} \\
& +\ln \int_{M} h e^{u_{t}^{(1)}-G_{t}}\left(1+u_{t}^{(2)}-u_{t}^{(1)}+O\left(\left|u_{t}^{(1)}-u_{t}^{(2)}\right|^{2}\right) d v_{g}\right. \\
= & u_{t}^{(1)}-u_{t}^{(2)}-\frac{\int_{M} h e^{u_{t}^{(1)}-G_{t}}\left(u_{t}^{(1)}-u_{t}^{(2)}\right) d v_{g}}{\int_{M} h e^{u_{t}^{(1)}-G_{t}} d v_{g}}+O\left(\left\|u_{t}^{(1)}-u_{t}^{(2)}\right\|_{L^{\infty}(M)}^{2}\right) .
\end{aligned}
$$

Let

$$
A_{t}:=\int_{B_{2 R_{0} t}\left(t p_{t}^{(1)}\right)} \frac{\rho h e^{-G_{t}}\left(e^{\widetilde{u}_{t}^{(1)}}-e^{\widetilde{u}_{t}^{(2)}}\right)}{\left\|u_{t}^{(1)}-u_{t}^{(2)}\right\|_{L^{\infty}(M)}} d x=\int_{B_{2 R_{0} t}\left(t p_{t}^{(1)}\right)}-\Delta \zeta_{t} d x
$$

Without loss of generality, from now on, we assume that

$$
\left.\nabla_{x}(8 \pi R(x, 0)+w(x))\right|_{x=0}=0 .
$$

Indeed, we can change the regular part of $G(x, 0)$ locally such that

$$
8 \pi R_{\text {new }}(x, 0)=8 \pi R_{\text {old }}(x, 0)-\left.\nabla_{x}\left(8 \pi R_{\text {old }}(x, 0)+w(x)\right)\right|_{x=0} \cdot x .
$$

Now we shall improve Lemma 3.6-(ii) by applying the arguments in [34].

\section{Lemma 3.7.}

$$
A_{t}=\int_{B_{2 R_{0} t}\left(t p_{t}^{(1)}\right)}-\Delta \zeta_{t} d x=O(t)
$$

Proof. Recall that

$$
\begin{aligned}
v_{t}^{(i)}(y) & =\widetilde{u}_{t}^{(i)}(t y)+6 \ln t-\bar{\varphi}(t y) \\
& =\eta_{t}^{(i)}(y)+I_{t}^{(i)}(y)+G_{*, t}^{(i)}(t y)-G_{*, t}^{(i)}\left(t p_{t}^{(i)}\right) \text { for } i=1,2 .
\end{aligned}
$$

Set

$$
\widetilde{v}_{t}^{(i)}(z)=v_{t}^{(i)}\left(\Lambda_{t,-}^{(1)} z+p_{t}^{(1)}\right) \text { for } i=1,2
$$

Then

$$
\begin{aligned}
\widetilde{v}_{t}^{(i)}(z)= & \eta_{t}^{(i)}\left(\Lambda_{t,-}^{(1)} z+p_{t}^{(1)}\right)+\ln \frac{e^{\lambda_{t}^{(i)}}}{\left.\left.\left(1+\left(\Lambda_{t,+}^{(i)}\right)^{2} \mid \Lambda_{t,-}^{(1)} z+p_{t}^{(1)}-q_{t}^{(i)}\right)\right|^{2}\right)^{2}} \\
& +G_{*, t}^{(i)}\left(t \Lambda_{t,-}^{(1)} z+t p_{t}^{(1)}\right)-G_{*, t}^{(i)}\left(t p_{t}^{(i)}\right) .
\end{aligned}
$$


We also see from (3.48) that

$$
\begin{aligned}
\frac{\widetilde{v}_{t}^{(1)}(z)-\widetilde{v}_{t}^{(2)}(z)}{\left\|u_{t}^{(1)}-u_{t}^{(2)}\right\|_{L \infty(M)}} & =\frac{\widetilde{u}_{t}^{(1)}\left(t \Lambda_{t,-}^{(1)} z+t p_{t}^{(1)}\right)-\widetilde{u}_{t}^{(2)}\left(t \Lambda_{t,-}^{(1)} z+t p_{t}^{(1)}\right)}{\left\|u_{t}^{(1)}-u_{t}^{(2)}\right\|_{L \infty(M)}} \\
& =\widetilde{\zeta}_{t}(z)+O\left(\left\|u_{t}^{(1)}-u_{t}^{(2)}\right\|_{L^{\infty}(M)}\right),
\end{aligned}
$$

which implies

$$
\begin{aligned}
\frac{1-e^{\widetilde{v}_{t}^{(2)}-\widetilde{v}_{t}^{(1)}}}{\left\|u_{t}^{(1)}-u_{t}^{(2)}\right\|_{L \infty(M)}} & =\frac{\widetilde{v}_{t}^{(1)}(z)-\widetilde{v}_{t}^{(2)}(z)+O\left(\left|\widetilde{v}_{t}^{(1)}-\widetilde{v}_{t}^{(2)}\right|^{2}\right)}{\left\|u_{t}^{(1)}-u_{t}^{(2)}\right\|_{L \infty(M)}} \\
& =\widetilde{\zeta}_{t}(z)+O\left(\left\|u_{t}^{(1)}-u_{t}^{(2)}\right\|_{L^{\infty}(M)}\right) .
\end{aligned}
$$

We have

$$
\Delta_{z} \widetilde{v}_{t}^{(i)}(z)+\left(\Lambda_{t,-}^{(1)}\right)^{2} h_{t}\left(\Lambda_{t,-}^{(1)} z+p_{t}^{(1)}\right) e^{\widetilde{v}_{t}^{(i)}(z)}=0
$$

where $h_{t}(y)=\rho h(t y)|y-\underline{e}|^{2}|y+\underline{e}|^{2} e^{-R_{t}(t y)+\psi(t y)}$. We see that

$$
\begin{aligned}
& \left(\Delta\left(\widetilde{v}_{t}^{(1)}-\widetilde{v}_{t}^{(2)}\right)\right)\left(\nabla\left(\widetilde{v}_{t}^{(1)}+\widetilde{v}_{t}^{(2)}\right) \cdot z\right)+\left(\Delta\left(\widetilde{v}_{t}^{(1)}+\widetilde{v}_{t}^{(2)}\right)\right)\left(\nabla\left(\widetilde{v}_{t}^{(1)}-\widetilde{v}_{t}^{(2)}\right) \cdot z\right) \\
& =\operatorname{div}\left\{\left(\nabla\left(\widetilde{v}_{t}^{(1)}-\widetilde{v}_{t}^{(2)}\right)\right)\left(\nabla\left(\widetilde{v}_{t}^{(1)}+\widetilde{v}_{t}^{(2)}\right) \cdot z\right)\right. \\
& \left.+\left(\nabla\left(\widetilde{v}_{t}^{(1)}+\widetilde{v}_{t}^{(2)}\right)\right)\left(\nabla\left(\widetilde{v}_{t}^{(1)}-\widetilde{v}_{t}^{(2)}\right) \cdot z\right)-\nabla\left(\widetilde{v}_{t}^{(1)}-\widetilde{v}_{t}^{(2)}\right) \cdot \nabla\left(\widetilde{v}_{t}^{(1)}+\widetilde{v}_{t}^{(2)}\right) z\right\},
\end{aligned}
$$

and

$$
\begin{aligned}
& \left(\Delta\left(\widetilde{v}_{t}^{(1)}-\widetilde{v}_{t}^{(2)}\right)\right)\left(\nabla\left(\widetilde{v}_{t}^{(1)}+\widetilde{v}_{t}^{(2)}\right) \cdot z\right)+\left(\Delta\left(\widetilde{v}_{t}^{(1)}+\widetilde{v}_{t}^{(2)}\right)\right)\left(\nabla\left(\widetilde{v}_{t}^{(1)}-\widetilde{v}_{t}^{(2)}\right) \cdot z\right) \\
& =-\left(\Lambda_{t,-}^{(1)}\right)^{2} h_{t}\left(\Lambda_{t,-}^{(1)} z+p_{t}^{(1)}\right)\left(e^{\widetilde{v}_{t}^{(1)}(z)}-e^{\widetilde{v}_{t}^{(2)}(z)}\right)\left(\nabla\left(\widetilde{v}_{t}^{(1)}+\widetilde{v}_{t}^{(2)}\right) \cdot z\right) \\
& -\left(\Lambda_{t,-}^{(1)}\right)^{2} h_{t}\left(\Lambda_{t,-}^{(1)} z+p_{t}^{(1)}\right)\left(e^{\widetilde{v}_{t}^{(1)}(z)}+e^{\widetilde{v}_{t}^{(2)}(z)}\right)\left(\nabla\left(\widetilde{v}_{t}^{(1)}-\widetilde{v}_{t}^{(2)}\right) \cdot z\right) \\
& =-\operatorname{div}\left(2\left(\Lambda_{t,-}^{(1)}\right)^{2} h_{t}\left(\Lambda_{t,-}^{(1)} z+p_{t}^{(1)}\right)\left(e^{\widetilde{v}_{t}^{(1)}(z)}-e^{\widetilde{v}_{t}^{(2)}(z)}\right) z\right) \\
& +4\left(\Lambda_{t,-}^{(1)}\right)^{2} h_{t}\left(\Lambda_{t,-}^{(1)} z+p_{t}^{(1)}\right)\left(e^{\widetilde{v}_{t}^{(1)}(z)}-e^{\widetilde{v}_{t}^{(2)}(z)}\right) \\
& +2\left(\Lambda_{t,-}^{(1)}\right)^{2} h_{t}\left(\Lambda_{t,-}^{(1)} z+p_{t}^{(1)}\right)\left(e^{\widetilde{v}_{t}^{(1)}(z)}-e^{\widetilde{v}_{t}^{(2)}(z)}\right)\left(\nabla_{z} \ln h_{t}\left(\Lambda_{t,-}^{(1)} z+p_{t}^{(1)}\right) \cdot z\right)
\end{aligned}
$$

Therefore, we obtain for any $r>0$, 


$$
\begin{aligned}
& \frac{1}{2} \int_{\partial B_{r}(0)} \nabla\left(\widetilde{v}_{t}^{(1)}-\widetilde{v}_{t}^{(2)}\right) \cdot \nabla\left(\widetilde{v}_{t}^{(1)}+\widetilde{v}_{t}^{(2)}\right)|z| d \sigma \\
& \quad-\int_{\partial B_{r}(0)} \frac{\left(\nabla\left(\widetilde{v}_{t}^{(1)}-\widetilde{v}_{t}^{(2)}\right) \cdot z\right)\left(\nabla\left(\widetilde{v}_{t}^{(1)}+\widetilde{v}_{t}^{(2)}\right) \cdot z\right)}{|z|} d \sigma \\
& =\int_{\partial B_{r}(0)}\left(\Lambda_{t,-}^{(1)}\right)^{2} h_{t}\left(\Lambda_{t,-}^{(1)} z+p_{t}^{(1)}\right) e^{\widetilde{v}_{t}^{(1)}(z)}\left(1-e^{\widetilde{v}_{t}^{(2)}(z)-\widetilde{v}_{t}^{(1)}(z)}\right)|z| d \sigma \\
& -\int_{B_{r}(0)}\left(\Lambda_{t,-}^{(1)}\right)^{2} h_{t}\left(\Lambda_{t,-}^{(1)} z+p_{t}^{(1)}\right) e^{\widetilde{v}_{t}^{(1)}(z)}\left(1-e^{\widetilde{v}_{t}^{(2)}(z)-\widetilde{v}_{t}^{(1)}(z)}\right) \\
& \quad \times\left(2+\nabla_{z} \ln h_{t}\left(\Lambda_{t,-}^{(1)} z+p_{t}^{(1)}\right) \cdot z\right) d z .
\end{aligned}
$$

Let $2 R_{0} \Lambda_{t,+}^{(1)} \leq|z| \leq \frac{r_{0}}{t} \Lambda_{t,+}^{(1)}$. By (2.12) and Theorem D, we have

$$
\nabla_{z} \bar{\varphi}\left(t \Lambda_{t,-}^{(1)} z+t p_{t}^{(1)}\right)=t^{2} O\left(t \Lambda_{t,-}^{(1)} z+t p_{t}^{(1)}\right)=O\left(t^{4}(|z|+1)\right)
$$

and

$$
\begin{aligned}
\nabla_{z} \widetilde{v}_{t}^{(i)}(z)= & \nabla_{z}\left(\widetilde{u}_{t}^{(i)}\left(t \Lambda_{t,-}^{(1)} z+t p_{t}^{(1)}\right)-\bar{\varphi}\left(t \Lambda_{t,-}^{(1)} z+t p_{t}^{(1)}\right)\right) \\
= & \nabla_{z} \widetilde{\phi}_{t}^{(i)}\left(t \Lambda_{t,-}^{(1)} z+t p_{t}^{(1)}\right) \\
& +\nabla_{z}\left(\rho_{t}^{(i)} G\left(t \Lambda_{t,-}^{(1)} z+t p_{t}^{(1)}, t p_{t}^{(i)}\right)+w\left(t \Lambda_{t,-}^{(1)} z+t p_{t}^{(1)}\right)\right)+O\left(t^{4}|z|\right) \\
= & \nabla_{z} \widetilde{\phi}_{t}^{(i)}\left(t \Lambda_{t,-}^{(1)} z+t p_{t}^{(1)}\right)-\frac{\rho_{t}^{(i)} t \Lambda_{t,-}^{(1)}}{2 \pi} \frac{\left(t \Lambda_{t,-}^{(1)} z+t p_{t}^{(1)}-t p_{t}^{(i)}\right)}{\left|t \Lambda_{t,-}^{(1)} z+t p_{t}^{(1)}-t p_{t}^{(i)}\right|^{2}} \\
+ & \nabla_{z}\left(\rho_{t}^{(i)} R\left(t \Lambda_{t,-}^{(1)} z+t p_{t}^{(1)}, t p_{t}^{(i)}\right)+w\left(t \Lambda_{t,-}^{(1)} z+t p_{t}^{(1)}\right)\right)+O\left(t^{4}|z|\right) \\
= & -\frac{\rho_{t}^{(i)}}{2 \pi} \frac{\left(z+\Lambda_{t,+}^{(1)}\left(p_{t}^{(1)}-p_{t}^{(i)}\right)\right)}{\left|z+\Lambda_{t,+}^{(1)}\left(p_{t}^{(1)}-p_{t}^{(i)}\right)\right|^{2}}+O\left(t^{2}\left\|\nabla \widetilde{\phi}_{t}\right\|_{L^{\infty}\left(M \backslash B_{2} R_{0}\left(t p_{t}^{(1)}\right)\right)}\right)+O\left(t^{4}|z|\right) \\
+ & \left.O\left(t^{2}\right) \nabla_{x}\left(\rho_{t}^{(i)} R\left(x, t p_{t}^{(i)}\right)+w(x)\right)\right|_{x=t \Lambda_{t,-}^{(1)} z+t p_{t}^{(1)}}
\end{aligned}
$$

In view of Lemma 3.1 and Theorem $\mathrm{D}$, we see that there are $a_{t}^{(i)} \in \mathbb{R}^{2}$ such that $a_{t}^{(1)}=0$, $\left|a_{t}^{(2)}\right|=O(t \ln t)$, and

$$
\begin{aligned}
\nabla_{z} \widetilde{v}_{t}^{(i)}(z) & =-4 \frac{z+a_{t}^{(i)}}{\left|z+a_{t}^{(i)}\right|^{2}}+O\left(\left.t^{2} \nabla_{x}\left(\rho_{t}^{(1)} R\left(x, t p_{t}^{(1)}\right)+w(x)\right)\right|_{x=t p_{t}^{(1)}}\right) \\
& +O\left(t^{3} \ln t\right)+O\left(t^{4}|z|\right) \quad \text { for } 2 R_{0} \Lambda_{t,+}^{(1)} \leq|z| \leq \frac{r_{0}}{t} \Lambda_{t,+}^{(1)} .
\end{aligned}
$$


In view of (3.50) and Theorem $\mathrm{D}$, we have $\left.\nabla_{x}\left(\rho_{t}^{(1)} R\left(x, t p_{t}^{(1)}\right)+w(x)\right)\right|_{x=t p_{t}^{(1)}}=O\left(t^{2} \ln t\right)$, and get that if $2 R_{0} \Lambda_{t,+}^{(1)} \leq|z| \leq \frac{r_{0}}{t} \Lambda_{t,+}^{(1)}$, then

$$
\begin{aligned}
\nabla_{z} \widetilde{v}_{t}^{(i)}(z) & =-4 \frac{\left(z+a_{t}^{(i)}\right)}{\left|z+a_{t}^{(i)}\right|^{2}}+O\left(t^{3} \ln t\right)+O\left(t^{4}|z|\right) \\
& =-4 \frac{z}{|z|^{2}}+O\left(\frac{\left|a_{t}^{(i)}\right|}{|z|^{2}}\right)+O\left(t^{3} \ln t\right)+O\left(t^{4}|z|\right) \\
& =-4 \frac{z}{|z|^{2}}+O\left(t^{3} \ln t\right)+O\left(t^{4}|z|\right)
\end{aligned}
$$

From (3.34), we recall $\int_{M} \zeta_{t} d v_{g}=0$. Together with Green's representation formula, we have

$$
\zeta_{t}(x)=\int_{M} \rho h e^{-G_{t}} \frac{\left(e^{\widetilde{u}_{t}^{(1)}}-e^{\widetilde{u}_{t}^{(2)}}\right)}{\left\|u_{t}^{(1)}-u_{t}^{(2)}\right\|_{L^{\infty}(M)}} G(x, y) d y
$$

and thus

$$
\begin{aligned}
\nabla_{x} \zeta_{t}(x) & =\int_{M \backslash B_{2 R_{0} t}\left(t p_{t}^{(1)}\right)} \rho h e^{-G_{t}} \frac{\left(e^{\widetilde{u}_{t}^{(1)}}-e^{\widetilde{u}_{t}^{(2)}}\right)}{\left\|u_{t}^{(1)}-u_{t}^{(2)}\right\|_{L^{\infty}(M)}} \nabla_{x} G(x, y) d y \\
& +\nabla_{x} G\left(x, t p_{t}^{(1)}\right) \int_{B_{2 R_{0} t}\left(t p_{t}^{(1)}\right)} \rho h e^{-G_{t}} \frac{\left(e^{\widetilde{u}_{t}^{(1)}}-e^{\widetilde{u}_{t}^{(2)}}\right)}{\left\|u_{t}^{(1)}-u_{t}^{(2)}\right\|_{L^{\infty}(M)}} d y \\
& +\int_{B_{2 R_{0} t}\left(t p_{t}^{(1)}\right)} \rho h e^{-G_{t}} \frac{\left(e^{\widetilde{u}_{t}^{(1)}}-e^{\left.\widetilde{u}_{t}^{(2)}\right)}\right.}{\left\|u_{t}^{(1)}-u_{t}^{(2)}\right\|_{L^{\infty}(M)}}\left(\nabla_{x} G(x, y)-\nabla_{x} G\left(x, t p_{t}^{(1)}\right)\right) d y \\
& :=I+I I+I I I .
\end{aligned}
$$

From Lemma 3.4-(ii) and Lemma 3.6-(iii), we see that if $x \in M \backslash B_{2 R_{0} t}\left(t p_{t}^{(1)}\right)$, then

$$
\begin{aligned}
I= & \int_{M \backslash B_{2 R_{0} t}\left(t p_{t}^{(1)}\right)} \nabla_{x} G(x, y) \frac{\rho h e^{-G_{t}+\widetilde{u}_{t}^{(1)}}}{\left\|u_{t}^{(1)}-u_{t}^{(2)}\right\|_{L^{\infty}(M)}} \\
& \times\left(u_{t}^{(1)}-u_{t}^{(2)}-\frac{\int_{M} h e^{u_{t}^{(1)}-G_{t}}\left(u_{t}^{(1)}-u_{t}^{(2)}\right) d v_{g}}{\int_{M} h e^{u_{t}^{(1)}-G_{t}} d v_{g}}+O\left(\left\|u_{t}^{(1)}-u_{t}^{(2)}\right\|_{L^{\infty}(M)}^{2}\right)\right) d y \\
= & \int_{M \backslash B_{2 R_{0} t}\left(t p_{t}^{(1)}\right)} \nabla_{x} G(x, y) \rho h e^{-G_{t}+\widetilde{u}_{t}^{(1)}}
\end{aligned}
$$




$$
\begin{aligned}
& \times\left(\zeta_{t}-\frac{\int_{M} h e^{u_{t}^{(1)}-G_{t}} \zeta_{t} d v_{g}}{\int_{M} h e^{u_{t}^{(1)}-G_{t}} d v_{g}}+O\left(\left\|u_{t}^{(1)}-u_{t}^{(2)}\right\|_{L^{\infty}(M)}\right)\right) d y \\
& =o(1) \quad \text { as } t \rightarrow 0 .
\end{aligned}
$$

From Lemma 3.6-(ii), we have

$$
A_{t}=-\int_{B_{2 R_{0} t}\left(t p_{t}^{(1)}\right)} \Delta \zeta_{t} d x=-\int_{B_{2 R_{0} \Lambda_{t,+}^{(1)}}(0)} \Delta \widetilde{\zeta}_{t} d z=O(t \ln t)
$$

Then we see that if $x \in M \backslash B_{2 R_{0} t}\left(t p_{t}^{(1)}\right)$, then

$$
I I=\nabla_{x} G\left(x, t p_{t}^{(1)}\right) A_{t}=\left\{-\frac{1}{2 \pi} \frac{\left(x-t p_{t}^{(1)}\right) 1_{B_{r_{0}}\left(t p_{t}^{(1)}\right)}(x)}{\left|x-t p_{t}^{(1)}\right|^{2}}+O(1)\right\} A_{t}
$$

where

$$
1_{B_{r_{0}}\left(t p_{t}^{(1)}\right)}(x)=\left\{\begin{array}{lll}
1 & \text { if } & x \in B_{r_{0}}\left(t p_{t}^{(1)}\right) \\
0 & \text { if } & x \in M \backslash B_{r_{0}}\left(t p_{t}^{(1)}\right)
\end{array}\right.
$$

Now we also see that if $x \in M \backslash B_{2 R_{0} t}\left(t p_{t}^{(1)}\right)$, then

$$
\begin{aligned}
I I I & =\int_{B_{2 R_{0} t}\left(t p_{t}^{(1)}\right)} \frac{\rho h e^{-G_{t}}\left(e^{\widetilde{u}_{t}^{(1)}}-e^{\widetilde{u}_{t}^{(2)}}\right)}{2 \pi\left\|u_{t}^{(1)}-u_{t}^{(2)}\right\|_{L^{\infty}(M)}}\left(\frac{x-t p_{t}^{(1)}}{\left|x-t p_{t}^{(1)}\right|^{2}}-\frac{x-y}{|x-y|^{2}}\right) 1_{B_{r_{0}}\left(t p_{t}^{(1)}\right)}(x) d y+o(1) \\
& =-\frac{1}{2 \pi} \int_{B_{2 R_{0} t}\left(t p_{t}^{(1)}\right)} \Delta \zeta_{t}\left(\frac{x-t p_{t}^{(1)}}{\left|x-t p_{t}^{(1)}\right|^{2}}-\frac{x-y}{|x-y|^{2}}\right) 1_{B_{r_{0}}\left(t p_{t}^{(1)}\right)}(x) d y+o(1),
\end{aligned}
$$

and

$$
\begin{aligned}
& \int_{B_{2 R_{0} t}\left(t p_{t}^{(1)}\right)} \Delta \zeta_{t}\left(\frac{x-t p_{t}^{(1)}}{\left|x-t p_{t}^{(1)}\right|^{2}}-\frac{x-y}{|x-y|^{2}}\right) 1_{B_{r_{0}}\left(t p_{t}^{(1)}\right)}(x) d y \\
= & \left(\int_{B_{R_{0} \Lambda_{t,+}^{(1)}(0)}+} \int_{B_{2 R_{0} \Lambda_{t,+}^{(1)}}(0) \backslash B_{R_{0} \Lambda_{t,+}^{(1)}}(0)}\right) \Delta_{z} \widetilde{\zeta}_{t}(z) \\
\times & \left(\frac{x-t p_{t}^{(1)}}{\left|x-t p_{t}^{(1)}\right|^{2}}-\frac{x-t p_{t}^{(1)}-t \Lambda_{t,-}^{(1)} z}{\left|x-t p_{t}^{(1)}-t \Lambda_{t,-}^{(1)} z\right|^{2}}\right) 1_{B_{r_{0}}\left(t p_{t}^{(1)}\right)}(x) d z
\end{aligned}
$$




$$
\begin{aligned}
= & \int_{B_{R_{0} \Lambda_{t,+}^{(1)}}(0)}\left|\Delta_{z} \widetilde{\zeta}_{t}(z)\right| O\left(\frac{t \Lambda_{t,-}^{(1)}|z|}{\left|x-t p_{t}^{(1)}\right|^{2}}\right) 1_{B_{r_{0}}\left(t p_{t}^{(1)}\right)}(x) d z \\
+ & \int_{B_{2 R_{0} \Lambda_{t,+}^{(1)}}(0) \backslash B_{R_{0} \Lambda_{t,+}^{(1)}}(0)} \frac{O(1)}{|z|^{4}}\left(\frac{1}{\left|x-t p_{t}^{(1)}\right|}+\frac{1}{\left|x-t p_{t}^{(1)}-t \Lambda_{t,-}^{(1)} z\right|}\right) 1_{B_{r_{0}}\left(t p_{t}^{(1)}\right)}(x) d z \\
= & \int_{B_{R_{0} \Lambda_{t,+}^{(1)}}(0)} O\left(\frac{1}{\left(1+|z|^{2}\right)^{2}}\right)\left(\frac{t^{2}|z|}{\left|x-t p_{t}^{(1)}\right|^{2}}\right) 1_{B_{r_{0}}\left(t p_{t}^{(1)}\right)}(x) d z \\
+ & \int_{2 R_{0} \Lambda_{t,+}^{(1)}(0) \backslash B_{R_{0} \Lambda_{t,+}^{(1)}}(0)} \frac{O(1)}{|z|^{4}}\left(O\left(t^{-1}\right)+\frac{O\left(t^{-2}\right)}{\left|\frac{x-t p_{t}^{(1)}}{t \Lambda_{t,-}^{(1)}}-z\right|}\right) 1_{B_{r_{0}}\left(t p_{t}^{(1)}\right)}(x) d z \\
= & O\left(\frac{t^{2} 1_{B_{r_{0}}\left(t p_{t}^{(1)}\right)}(x)}{\left|x-t p_{t}^{(1)}\right|^{2}}\right)+o(1) \text { as } t \rightarrow 0 .
\end{aligned}
$$

From (3.64)-(3.68), we see that if $x \in M \backslash B_{2 R_{0} t}\left(t p_{t}^{(1)}\right)$,

$$
\nabla_{x} \zeta_{t}(x)=-\frac{A_{t}}{2 \pi} \frac{\left(x-t p_{t}^{(1)}\right) 1_{B_{r_{0}}\left(t p_{t}^{(1)}\right)}(x)}{\left|x-t p_{t}^{(1)}\right|^{2}}+O\left(\frac{t^{2} 1_{B_{r_{0}}\left(t p_{t}^{(1)}\right)}(x)}{\left|x-t p_{t}^{(1)}\right|^{2}}\right)+o(1) \text { as } t \rightarrow 0
$$

Here we also note that if $2 R_{0} \Lambda_{t,+}^{(1)} \leq|z| \leq \frac{2 r_{0} \Lambda_{t,+}^{(1)}}{t}$, then

$$
\begin{aligned}
& \frac{\nabla_{z}\left(\widetilde{v}_{t}^{(1)}(z)-\widetilde{v}_{t}^{(2)}(z)\right)}{\left\|u_{t}^{(1)}-u_{t}^{(2)}\right\|_{L^{\infty}(M)}} \\
& =\frac{\nabla_{z}\left(\widetilde{u}_{t}^{(1)}\left(t \Lambda_{t,-}^{(1)} z+t p_{t}^{(1)}\right)-\widetilde{u}_{t}^{(2)}\left(t \Lambda_{t,-}^{(1)} z+t p_{t}^{(1)}\right)\right)}{\left\|u_{t}^{(1)}-u_{t}^{(2)}\right\|_{L^{\infty}(M)}} \\
& =\nabla_{z} \zeta_{t}\left(t \Lambda_{t,-}^{(1)} z+t p_{t}^{(1)}\right) \\
& =\nabla_{z} \widetilde{\zeta}_{t}(z)=t \Lambda_{t,-}^{(1)}\left(-\frac{A_{t}}{2 \pi} \frac{1}{t \Lambda_{t,-}^{(1)}} \frac{z}{|z|^{2}}+O(1)\right)=-\frac{A_{t}}{2 \pi} \frac{z}{|z|^{2}}+O\left(t^{2}\right),
\end{aligned}
$$

and

$$
\left.\nabla_{z} \widetilde{\zeta}_{t}(z)\right|_{z \in \partial B_{2 R_{0} \Lambda_{t,+}^{(1)}}^{(0)}}=\left.t \Lambda_{t,-}^{(1)} \nabla_{x} \zeta_{t}(x)\right|_{x \in \partial B_{2 R_{0} t}\left(t p_{t}^{(1)}\right)}
$$

By (3.59), we obtain for $r_{t, R_{0}}=2 R_{0} \Lambda_{t,+}^{(1)}$, 


$$
\begin{aligned}
& \frac{1}{2} \int_{\partial B_{r_{t, R_{0}}}(0)} \nabla\left(\widetilde{\zeta}_{t}\right) \cdot \nabla\left(\widetilde{v}_{t}^{(1)}+\widetilde{v}_{t}^{(2)}\right)|z| d \sigma-\int_{\partial B_{r_{t, R_{0}}}(0)} \frac{\left(\nabla\left(\widetilde{\zeta}_{t}\right) \cdot z\right)\left(\nabla\left(\widetilde{v}_{t}^{(1)}+\widetilde{v}_{t}^{(2)}\right) \cdot z\right)}{|z|} d \sigma \\
& =\int_{\partial B_{r_{t, R_{0}}}(0)} \frac{\left(\Lambda_{t,-}^{(1)}\right)^{2} h_{t}\left(\Lambda_{t,-}^{(1)} z+p_{t}^{(1)}\right)}{\left\|u_{t}^{(1)}-u_{t}^{(2)}\right\|_{L^{\infty}(M)}} e^{\widetilde{v}_{t}^{(1)}(z)}\left(1-e^{\widetilde{v}_{t}^{(2)}(z)-\widetilde{v}_{t}^{(1)}(z)}\right)|z| d \sigma \\
& -\int_{B_{r_{t}, R_{0}}(0)} \frac{\left(\Lambda_{t,-}^{(1)}\right)^{2} h_{t}\left(\Lambda_{t,-}^{(1)} z+p_{t}^{(1)}\right)}{\left\|u_{t}^{(1)}-u_{t}^{(2)}\right\|_{L^{\infty}(M)}} e^{\widetilde{v}_{t}^{(1)}(z)}\left(1-e^{\widetilde{v}_{t}^{(2)}(z)-\widetilde{v}_{t}^{(1)}(z)}\right) \\
& \times\left(2+\nabla_{z} \ln h_{t}\left(\Lambda_{t,-}^{(1)} z+p_{t}^{(1)}\right) \cdot z\right) d z .
\end{aligned}
$$

We see from (3.62) and (3.70) that

$$
\begin{aligned}
& (\mathrm{LHS}) \text { of }(3.72)=\frac{1}{2} \int_{\partial B_{r_{t, R_{0}}}(0)}|z|\left(-8 \frac{z}{|z|^{2}}+O\left(t^{3} \ln t\right)\right) \cdot\left(-\frac{A_{t}}{2 \pi} \frac{z}{|z|^{2}}+O\left(t^{2}\right)\right) d \sigma \\
& -\int_{\partial B_{r_{t, R_{0}}}(0)} \frac{1}{|z|}\left(-\frac{A_{t}}{2 \pi}+O(t)\right)\left(-8+O\left(t^{2} \ln t\right)\right) d \sigma \\
& =\frac{1}{2} \int_{\partial B_{r_{t}, R_{0}}(0)}|z|\left(-8 \frac{z}{|z|^{2}}+O\left(t^{3} \ln t\right)\right) \cdot\left(-\frac{A_{t}}{2 \pi} \frac{z}{|z|^{2}}+O\left(t^{2}\right)\right) d \sigma \\
& -\int_{\partial B_{r_{t, R_{0}}}(0)} \frac{1}{|z|}\left(\frac{4 A_{t}}{\pi}+O(t)\right) d \sigma \\
& =\frac{1}{2} \int_{\partial B_{r_{t, R_{0}}}(0)}|z|\left(\frac{4 A_{t}}{\pi|z|^{2}}+O\left(t^{3}\right)\right) d \sigma-\int_{\partial B_{r_{t, R_{0}}}(0)} \frac{1}{|z|}\left(\frac{4 A_{t}}{\pi}\right) d \sigma+O(t) \\
& =-4 A_{t}+O(t) \text {. }
\end{aligned}
$$

We also see from (3.55)-(3.56) and (3.53) that

$$
\begin{gathered}
(\mathrm{RHS}) \text { of }(3.72)=\int_{B_{r_{t, R_{0}}}(0)} \frac{2\left(\Delta \widetilde{v}_{t}^{(1)}-\Delta \widetilde{v}_{t}^{(2)}\right)}{\left\|u_{t}^{(1)}-u_{t}^{(2)}\right\|_{L^{\infty}(M)}}+\frac{O(t|z|)}{\left(1+|z|^{2}\right)^{2}} d z+\int_{\partial B_{r_{t, R_{0}}}(0)} O\left(\frac{1}{|z|^{3}}\right) d \sigma \\
=2 \int_{B_{r_{t, R_{0}}}(0)} \Delta_{z} \widetilde{\zeta}_{t}(z) d z+O(t)
\end{gathered}
$$




$$
=2 \int_{B_{2 R_{0} t}\left(t p_{t}^{(1)}\right)} \Delta_{x} \zeta_{t}(x) d x+O(t)=-2 A_{t}+O(t) .
$$

By (3.73)-(3.74), we obtain $A_{t}=O(t)$, and complete the proof of Lemma 3.7.

For any function $f$, we denote

$$
D_{l} f(z)=\frac{\partial f(z)}{\partial z_{l}} \text { for } l=1,2
$$

Lemma 3.8. (i) $b_{1}=b_{2}=0$,

$$
\begin{aligned}
& \text { (ii) } \widetilde{\zeta}_{t}(z) \rightarrow 0, \zeta_{t}\left(t \Lambda_{t,-}^{(1)} z+t p_{t}^{(1)}\right) \rightarrow 0 \text { in } C_{l o c}^{0}\left(\mathbb{R}^{2}\right) \text { as } t \rightarrow 0, \\
& \text { (iii) } \lim _{t \rightarrow 0}\left(\int_{\left.B_{2 R_{0} \Lambda_{t,+}^{(1)}(0)}(\ln |z|) \Delta \widetilde{\zeta}_{t} d z\right)=0 .}\right.
\end{aligned}
$$

Proof. (i) We have

$$
\begin{aligned}
& \operatorname{div}\left(\nabla \widetilde{\zeta}_{t} D_{l} \widetilde{v}_{t}^{(i)}+\nabla \widetilde{v}_{t}^{(i)} D_{l} \widetilde{\zeta}_{t}-\nabla \widetilde{\zeta}_{t} \cdot \nabla \widetilde{v}_{t}^{(i)} e_{l}\right)=\Delta \widetilde{\zeta}_{t} D_{l} \widetilde{v}_{t}^{(i)}+\Delta \widetilde{v}_{t}^{(i)} D_{l} \widetilde{\zeta}_{t} \\
& =\frac{\Delta\left(\widetilde{v}_{t}^{(1)}-\widetilde{v}_{t}^{(2)}\right)}{\left\|u_{t}^{(1)}-u_{t}^{(2)}\right\|_{L^{\infty}(M)}} D_{l} \widetilde{v}_{t}^{(i)}+\frac{\Delta \widetilde{v}_{t}^{(i)} D_{l}\left(\widetilde{v}_{t}^{(1)}-\widetilde{v}_{t}^{(2)}\right)}{\left\|u_{t}^{(1)}-u_{t}^{(2)}\right\|_{L^{\infty}(M)}} \\
& =-\frac{\left(\Lambda_{t,-}^{(1)}\right)^{2} h_{t}\left(\Lambda_{t,-}^{(1)} z+p_{t}^{(1)}\right)\left(e^{\widetilde{v}_{t}^{(1)}}-e^{\widetilde{v}_{t}^{(2)}}\right) D_{l} \widetilde{v}_{t}^{(i)}}{\left\|u_{t}^{(1)}-u_{t}^{(2)}\right\|_{L^{\infty}(M)}} \\
& -\frac{\left(\Lambda_{t,-}^{(1)}\right)^{2} h_{t}\left(\Lambda_{t,-}^{(1)} z+p_{t}^{(1)}\right) e^{\widetilde{v}_{t}^{(i)}} D_{l}\left(\widetilde{v}_{t}^{(1)}-\widetilde{v}_{t}^{(2)}\right)}{\left\|u_{t}^{(1)}-u_{t}^{(2)}\right\|_{L^{\infty}(M)}} \\
& =-\operatorname{div}\left(\frac{\left(\Lambda_{t,-}^{(1)}\right)^{2} h_{t}\left(\Lambda_{t,-}^{(1)} z+p_{t}^{(1)}\right)\left(e^{\widetilde{v}_{t}^{(1)}}-e^{\widetilde{v}_{t}^{(2)}}\right) e_{l}}{\left\|u_{t}^{(1)}-u_{t}^{(2)}\right\|_{L^{\infty}(M)}}\right) \\
& +\left[\frac{\left(\Lambda_{t,-}^{(1)}\right)^{2} h_{t}\left(\Lambda_{t,-}^{(1)} z+p_{t}^{(1)}\right)\left(e^{\widetilde{v}_{t}^{(1)}}-e^{\widetilde{v}_{t}^{(2)}}\right)}{\left\|u_{t}^{(1)}-u_{t}^{(2)}\right\|_{L^{\infty}(M)}} D_{l}\left((-1)^{i}\left(\widetilde{v}_{t}^{(1)}-\widetilde{v}_{t}^{(2)}\right)+\ln h_{t}\left(\Lambda_{t,-}^{(1)} z+p_{t}^{(1)}\right)\right)\right] \text {. }
\end{aligned}
$$

For any constant $R \geq R_{0}$, let $r_{t, R}=2 R \Lambda_{t,+}^{(1)}$. Then (3.76) implies

$$
\begin{aligned}
& \int_{\partial B_{r_{t, R}}(0)}\left(2 \nabla \widetilde{\zeta}_{t} D_{l} \widetilde{v}_{t}^{(1)}+2 \nabla \widetilde{v}_{t}^{(2)} D_{l} \widetilde{\zeta}_{t}-\nabla \widetilde{\zeta}_{t} \cdot\left(\nabla \widetilde{v}_{t}^{(1)}+\nabla \widetilde{v}_{t}^{(2)}\right) e_{l}\right) \cdot \frac{z}{|z|} d \sigma \\
=-2 & \int_{\partial B_{r_{t, R}(0)}}\left(\frac{\left(\Lambda_{t,-}^{(1)}\right)^{2} h_{t}\left(\Lambda_{t,-}^{(1)} z+p_{t}^{(1)}\right)\left(e^{(1)}-e^{(1)} \widetilde{v}_{t}^{(2)}\right) e_{l}}{\left\|u_{t}^{(1)}-u_{t}^{(2)}\right\|_{L^{\infty}(M)}}\right) \cdot \frac{z}{|z|} d \sigma
\end{aligned}
$$




$$
+2\left[\int_{B_{r_{t}, R}(0)} \frac{\left(\Lambda_{t,-}^{(1)}\right)^{2} h_{t}\left(\Lambda_{t,-}^{(1)} z+p_{t}^{(1)}\right)\left(e^{\widetilde{v}_{t}^{(1)}}-e^{\widetilde{v}_{t}^{(2)}}\right)}{\left\|u_{t}^{(1)}-u_{t}^{(2)}\right\|_{L^{\infty}(M)}} D_{l}\left(\ln h_{t}\left(\Lambda_{t,-}^{(1)} z+p_{t}^{(1)}\right)\right) d z\right]
$$

By (3.69) and Lemma 3.7, we have if $x \in M \backslash B_{2 R_{0} t}\left(t p_{t}^{(1)}\right)$, then

$$
\begin{aligned}
\nabla_{x} \zeta_{t}(x) & =-\frac{A_{t}\left(x-t p_{t}^{(1)}\right) 1_{B_{r_{0}}\left(t p_{t}^{(1)}\right)}(x)}{2 \pi\left|x-t p_{t}^{(1)}\right|^{2}}+\frac{O\left(t^{2}\right) 1_{B_{r_{0}}\left(t p_{t}^{(1)}\right)}(x)}{\left|x-t p_{t}^{(1)}\right|^{2}}+o(1) \\
& =\frac{O(t) 1_{B_{r_{0}}\left(t p_{t}^{(1)}\right)}(x)}{\left|x-t p_{t}^{(1)}\right|}+o(1)
\end{aligned}
$$

which implies

$$
\begin{aligned}
\nabla_{z} \widetilde{\zeta}_{t}(z) & =\left.t \Lambda_{t,-}^{(1)} \nabla_{x} \zeta_{t}(x)\right|_{x=t \Lambda_{t,-}^{(1)} z+t p_{t}^{(1)}} \\
& =\frac{O(t)}{|z|} 1_{B_{\frac{r_{0} \Lambda_{t,+}^{(1)}}{t}}(0)}(z)+o\left(t^{2}\right)
\end{aligned}
$$

for $2 R_{0} \Lambda_{t,+}^{(1)} \leq|z| \leq \frac{r_{0} \Lambda_{t,+}^{(1)}}{t}$.

Therefore, in view of (3.62) and (3.79) we get that

$$
\begin{aligned}
(\mathrm{LHS}) \text { of }(3.77) & =\left.|z|\left(O\left(t^{3} \ln t\right)+O\left(t^{4}|z|\right)+\frac{O(1)}{|z|}\right)\left(O\left(\frac{t}{|z|}\right)+o\left(t^{2}\right)\right)\right|_{z \in \partial B_{2 R \Lambda_{t,+}^{(1)}}(0)} \\
& =O\left(t^{4}(\ln t) R\right)+O\left(\frac{t^{2}}{R}\right)+o\left(t^{4}\right) R^{2}+o\left(t^{2}\right)
\end{aligned}
$$

To estimate (RHS) of (3.77), by the change of variables $x=t \Lambda_{t,-}^{(1)} z+t p_{t}^{(1)}$, we see that if $|z|=2 R \Lambda_{t,+}^{(1)} \geq 2 R_{0} \Lambda_{t,+}^{(1)}$, then Theorem D implies

$$
\begin{aligned}
& -2 \int_{\partial B_{r_{t, R}}(0)}\left(\frac{\left(\Lambda_{t,-}^{(1)}\right)^{2} h_{t}\left(\Lambda_{t,-}^{(1)} z+p_{t}^{(1)}\right)\left(e^{\widetilde{v}_{t}^{(1)}}-e^{\widetilde{v}_{t}^{(2)}}\right) e_{l}}{\left\|u_{t}^{(1)}-u_{t}^{(2)}\right\|_{L^{\infty}(M)}}\right) \cdot \frac{z}{|z|} d \sigma(z) \\
& =\frac{-2 \Lambda_{t,+}^{(1)}}{t} \int_{\partial B_{2 R t}\left(t p_{t}^{(1)}\right)} \frac{\left.\rho\left(\Lambda_{t,-}^{(1)}\right)^{2}\left(h(t y)|y-\underline{e}|^{2}|y+\underline{e}|^{2} e^{-R_{t}(t y)}\right)\right|_{y=\Lambda_{t,-}^{(1)} z+p_{t}^{(1)}}}{\left\|u_{t}^{(1)}-u_{t}^{(2)}\right\|_{L^{\infty}(M)}} \\
& \times \frac{\left(e^{\widetilde{u}_{t}^{(1)}}-e^{\left.\widetilde{u}_{t}^{(2)}\right) t^{6} e_{l} \cdot\left(x-t p_{t}^{(1)}\right)}\right.}{\left|x-t p_{t}^{(1)}\right|} d \sigma(x)
\end{aligned}
$$




$$
=O(1)\left(\int_{\partial B_{2 R t}\left(t p_{t}^{(1)}\right)} \frac{t^{2} h(x) e^{-G_{t}(x)}\left(e^{\widetilde{u}_{t}^{(1)}}-e^{\left.\widetilde{u}_{t}^{(2)}\right)} e_{l}\right.}{\left\|u_{t}^{(1)}-u_{t}^{(2)}\right\|_{L^{\infty}(M)}} \cdot \frac{x-t p_{t}^{(1)}}{\left|x-t p_{t}^{(1)}\right|} d \sigma(x)\right)=O\left(t^{3} R\right) .
$$

Let $x=t \Lambda_{t,-}^{(1)} z+t p_{t}^{(1)}$ and $y=\Lambda_{t,-}^{(1)} z+p_{t}^{(1)}$.

Then $r_{0} \geq\left|x-t p_{t}^{(1)}\right|=t \Lambda_{t,-}^{(1)}|z|=2 R t \geq 2 R_{0} t$ implies $\frac{r_{0}}{t} \geq\left|y-p_{t}^{(1)}\right|=\Lambda_{t,-}^{(1)}|z|=$ $2 R \geq 2 R_{0}$. So we see that if $2 R_{0} \leq\left|y-p_{t}^{(1)}\right| \leq \frac{r_{0}}{t}$, then

$$
\begin{aligned}
\nabla_{y} \ln h_{t}(y) & =\nabla_{y}\left(\ln \rho h(t y) e^{-R_{t}(t y)+\psi(t y)}+2 \ln |y-\underline{e}|+2 \ln |y+\underline{e}|\right) \\
& =t \nabla_{t y} \ln \left(\rho h(t y) e^{-R_{t}(t y)+\psi(t y)}\right)+O\left(\frac{1}{|y|}\right)=O(1)
\end{aligned}
$$

In view of Lemma 3.4-(ii) and Lemma 3.6-(iii), we have

$$
\zeta_{t}-\frac{\int_{M} h e^{u_{t}^{(1)}-G_{t}} \zeta_{t} d v_{g}}{\int_{M} h e^{u_{t}^{(1)}-G_{t}} d v_{g}}=o(1) \text { in } M \backslash B_{\frac{t R_{0}}{2}}\left(t p_{t}^{(1)}\right) .
$$

Together with (3.54), we see that

$$
\begin{aligned}
& \frac{1}{\left\|u_{t}^{(1)}-u_{t}^{(2)}\right\|_{L^{\infty}(M)}} \\
& \times \quad \int \quad\left(\Lambda_{t,-}^{(1)}\right)^{2} h_{t}\left(\Lambda_{t,-}^{(1)} z+p_{t}^{(1)}\right)\left(e^{\widetilde{v}_{t}^{(1)}}-e^{\widetilde{v}_{t}^{(2)}}\right) D_{l}\left(\ln h_{t}\left(\Lambda_{t,-}^{(1)} z+p_{t}^{(1)}\right)\right) d z \\
& B_{2 R \Lambda_{t,+}^{(1)}}(0) \backslash B_{2 R_{0} \Lambda_{t,+}^{(1)}}(0) \\
& =O\left(\frac{t^{3}}{\left\|u_{t}^{(1)}-u_{t}^{(2)}\right\|_{L^{\infty}(M)}}\right) \\
& \times\left.\int_{B_{2 R \Lambda_{t,+}^{(1)}}(0) \backslash B_{2 R_{0} \Lambda_{t,+}^{(1)}}(0)} h_{t}\left(\Lambda_{t,-}^{(1)} z+p_{t}^{(1)}\right)\left(e^{\widetilde{v}_{t}^{(1)}}-e^{\widetilde{v}_{t}^{(2)}}\right) D_{y_{l}} \ln h_{t}(y)\right|_{y=\Lambda_{t,-}^{(1)} z+p_{t}^{(1)}} d z \\
& =O\left(t^{3}\right) \quad \int_{B} h\left(t \Lambda_{t,-}^{(1)} z+t p_{t}^{(1)}\right) e^{-R_{t}\left(t \Lambda_{t,-}^{(1)} z+t p_{t}^{(1)}\right)} \\
& B_{2 R \Lambda_{t,+}^{(1)}}(0) \backslash B_{2 R_{0} \Lambda_{t,+}^{(1)}}(0) \\
& \times\left|\Lambda_{t,-}^{(1)} z+p_{t}^{(1)}-\underline{e}\right|^{2}\left|\Lambda_{t,-}^{(1)} z+p_{t}^{(1)}+\underline{e}\right|^{2} O\left(t^{6}\right) e^{\widetilde{u}_{t}^{(1)}\left(t \Lambda_{t,-}^{(1)} z+t p_{t}^{(1)}\right)} \\
& \times \frac{\left(\widetilde{u}_{t}^{(1)}\left(t \Lambda_{t,-}^{(1)} z+t p_{t}^{(1)}\right)-\widetilde{u}_{t}^{(2)}\left(t \Lambda_{t,-}^{(1)} z+t p_{t}^{(1)}\right)+O\left(\left\|u_{t}^{(1)}-u_{t}^{(2)}\right\|_{L^{\infty}(M)}^{2}\right)\right.}{\left\|u_{t}^{(1)}-u_{t}^{(2)}\right\|_{L^{\infty}(M)}} d z \\
& =O(t) \quad \quad \quad h(x)|x-t \underline{e}|^{2}|x+t \underline{e}|^{2} e^{-R_{t}(x)} e^{\widetilde{u}_{t}^{(1)}(x)} \\
& B_{2 R t}\left(t p_{t}^{(1)}\right) \backslash B_{2 R_{0} t}\left(t p_{t}^{(1)}\right)
\end{aligned}
$$




$$
\begin{aligned}
& \times\left(\zeta_{t}(x)-\frac{\int_{M} h e^{u_{t}^{(1)}-G_{t}} \zeta_{t} d v_{g}}{\int_{M} h e^{u_{t}^{(1)}-G_{t}} d v_{g}}+O\left(\left\|u_{t}^{(1)}-u_{t}^{(2)}\right\|_{L^{\infty}(M)}\right)\right) d x \\
& =O(t) \int_{B_{2 R t}\left(t p_{t}^{(1)}\right) \backslash B_{2 R_{0} t}\left(t p_{t}^{(1)}\right)} o(1) d x=o\left(t^{3}\right) R^{2} .
\end{aligned}
$$

To estimate $2 \int_{B_{2 R_{0} \Lambda_{t,+}^{(1)}}(0)} \frac{\left(\Lambda_{t,-}^{(1)}\right)^{2} h_{t}\left(\Lambda_{t,-}^{(1)} z+p_{t}^{(1)}\right)\left(e^{\tilde{v}_{t}^{(1)}}-e^{\widetilde{v}_{t}^{(2)}}\right) D_{l}\left(\ln h_{t}\left(\Lambda_{t,-}^{(1)} z+t p_{t}^{(1)}\right)\right)}{\left\|u_{t}^{(1)}-u_{t}^{(2)}\right\|_{L} \infty^{(M)}} d z$, we note that if $|z| \leq 2 R_{0} \Lambda_{t,+}^{(1)}$, then there is $\theta \in(0,1)$ such that

$$
\begin{aligned}
D_{z_{l}}\left[\ln h_{t}\left(\Lambda_{t,-}^{(1)} z+p_{t}^{(1)}\right)\right]=\left.\left[D_{y_{l}} \ln h_{t}(y)\right]\right|_{y=\Lambda_{t,-}^{(1)} z+p_{t}^{(1)}} \Lambda_{t,-}^{(1)} \\
=\left[\left.D_{y_{l}} \ln h_{t}(y)\right|_{y=p_{t}^{(1)}}+\left.\sum_{k=1}^{2} D_{y_{k}} D_{y_{l}} \ln h_{t}(y)\right|_{y=p_{t}^{(1)}} \Lambda_{t,-}^{(1)} z_{k}\right. \\
\left.+O\left(\left.D^{3} \ln h_{t}\right|_{y=\theta \Lambda_{t,-}^{(1)} z+p_{t}^{(1)}} t^{2}|z|^{2}\right)\right] \Lambda_{t,-}^{(1)} \\
=\left[\left.D_{y_{l}} \ln h_{t}(y)\right|_{y=p_{t}^{(1)}}+\left.\sum_{k=1}^{2} D_{y_{k}} D_{y_{l}} \ln h_{t}(y)\right|_{y=p_{t}^{(1)}} \Lambda_{t,-}^{(1)} z_{k}+O\left(t^{2}|z|^{2}\right)\right] \Lambda_{t,-}^{(1)} .
\end{aligned}
$$

Moreover, by using the proof of Lemma 3.1 and (3.50), we get that

$$
\left.\nabla_{y} \ln h_{t}(y)\right|_{y=p_{t}^{(1)}}=-\left.t \nabla_{x} G_{*, t}^{(1)}(x)\right|_{x=t p_{t}^{(1)}}+O\left(t\left\|\widetilde{\phi}_{t}\right\|_{*}+t^{2} \ln t\right)=O\left(t^{2} \ln t\right)
$$

Now we obtain

$$
\begin{aligned}
& D_{z_{l}}\left[\ln h_{t}\left(\Lambda_{t,-}^{(1)} z+p_{t}^{(1)}\right)\right] \\
& \quad=\left[\left.\sum_{k=1}^{2} D_{y_{k}} D_{y_{l}} \ln h_{t}(y)\right|_{y=p_{t}^{(1)}} \Lambda_{t,-}^{(1)} z_{k}+O\left(t^{2} \ln t\right)+O\left(t^{2}|z|^{2}\right)\right] \Lambda_{t,-}^{(1)} .
\end{aligned}
$$

Together with (3.53) and (3.55), we see that

$$
\begin{aligned}
& 2 \int_{B_{2 R_{0} \Lambda_{t,+}^{(1)}}(0)} \frac{\left(\Lambda_{t,-}^{(1)}\right)^{2} h_{t}\left(\Lambda_{t,-}^{(1)} z+p_{t}^{(1)}\right)\left(e^{\widetilde{v}_{t}^{(1)}}-e^{\widetilde{v}_{t}^{(2)}}\right)}{\left\|u_{t}^{(1)}-u_{t}^{(2)}\right\|_{L^{\infty}(M)}} D_{l}\left(\ln h_{t}\left(\Lambda_{t,-}^{(1)} z+p_{t}^{(1)}\right)\right) d z \\
& =2 \int_{\substack{B_{2 R_{0} \Lambda_{t,+}^{(1)}}(0)}} \frac{\left(\Lambda_{t,-}^{(1)}\right)^{2} h_{t}\left(\Lambda_{t,-}^{(1)} z+p_{t}^{(1)}\right) e^{\widetilde{v}_{t}^{(1)}}\left(1-e^{\left.\widetilde{v}_{t}^{(2)}-\widetilde{v}_{t}^{(1)}\right)}\right.}{\left\|u_{t}^{(1)}-u_{t}^{(2)}\right\|_{L^{\infty}(M)}}
\end{aligned}
$$




$$
\begin{aligned}
& \times\left[\left.\sum_{k=1}^{2} D_{y_{k}} D_{y_{l}} \ln h_{t}(y)\right|_{y=p_{t}^{(1)}} \Lambda_{t,-}^{(1)} z_{k}+O\left(t^{2} \ln t\right)+O\left(t^{2}|z|^{2}\right)\right] \Lambda_{t,-}^{(1)} d z \\
& =2\left\{\int_{B_{2 R_{0} \Lambda_{t,+}^{(1)}}(0)} \frac{h_{t}\left(\Lambda_{t,-}^{(1)} z+p_{t}^{(1)}\right)\left(1+\left|\widetilde{\eta}_{t}^{(1)}\right|+t^{2}|z|\right)}{C_{t}^{(1)}\left(1+\left|z+O\left(t^{2}\right)\right|^{2}\right)^{2}}\left(\widetilde{\zeta}_{t}(z)+O\left(\left\|u_{t}^{(1)}-u_{t}^{(2)}\right\|_{L^{\infty}(M)}\right)\right)\right. \\
& \left.\times\left[\left.\sum_{k=1}^{2} D_{y_{k}} D_{y_{l}} \ln h_{t}(y)\right|_{y=p_{t}^{(1)}} \Lambda_{t,-}^{(1)} z_{k}+O\left(t^{2} \ln t\right)+O\left(t^{2}|z|^{2}\right)\right] \Lambda_{t,-}^{(1)} d z\right\}
\end{aligned}
$$

We note from (3.86) that

$$
\begin{aligned}
\frac{h_{t}\left(\Lambda_{t,-}^{(1)} z+p_{t}^{(1)}\right)}{C_{t}^{(1)}} & =\frac{8 h_{t}\left(\Lambda_{t,-}^{(1)} z+p_{t}^{(1)}\right)}{h_{t}\left(p_{t}^{(1)}\right)}=8\left(1+\frac{\nabla h_{t}\left(p_{t}^{(1)}\right)}{h_{t}\left(p_{t}^{(1)}\right)} \cdot \Lambda_{t,-}^{(1)} z+O\left(t^{2}|z|^{2}\right)\right) \\
& =8+O\left(t^{2} \ln t\right)+O\left(t^{2}|z|^{2}\right) .
\end{aligned}
$$

By using Lemma 3.3 and Lemma 3.6-(v), we have

$$
\widetilde{\zeta}_{t} \rightarrow \sum_{i=1}^{2} \frac{b_{i} z_{i}}{1+|z|^{2}} \quad \text { in } \quad C_{\mathrm{loc}}^{0}\left(\mathbb{R}^{2}\right)
$$

Together with Theorem E, we have for any $R>1$,

$$
\begin{aligned}
& 2 \int_{B_{2 R_{0} \Lambda_{t,+}^{(1)}}(0)} \frac{\left(\Lambda_{t,-}^{(1)}\right)^{2} h_{t}\left(\Lambda_{t,-}^{(1)} z+p_{t}^{(1)}\right)\left(e^{\widetilde{v}_{t}^{(1)}}-e^{\widetilde{v}_{t}^{(2)}}\right)}{\left\|u_{t}^{(1)}-u_{t}^{(2)}\right\|_{L^{\infty}(M)}} D_{l}\left(\ln h_{t}\left(\Lambda_{t,-}^{(1)} z+t p_{t}^{(1)}\right)\right) d z \\
& =16 \int_{B_{R}(0)} \frac{\left(1+O\left(t^{2} \ln t(|z|+1)^{\varepsilon}\right)+O\left(t^{2}|z|^{2}\right)\right)}{\left(1+|z|^{2}\right)^{2}}\left(\sum_{i=1}^{2} \frac{b_{i} z_{i}}{\left(1+|z|^{2}\right)}+o(1)\right) \\
& \times\left[\left.\sum_{k=1}^{2} D_{y_{k}} D_{y_{l}} \ln h_{t}(y)\right|_{\left.y=p_{t}^{(1)} \Lambda_{t,-}^{(1)} z_{k}+O\left(t^{2} \ln t\right)+O\left(t^{2}|z|^{2}\right)\right] \Lambda_{t,-}^{(1)} d z} \frac{t}{|z|^{4}}\left(O(t|z|)+O\left(t^{2} \ln t\right)\right) d z\right. \\
& +\int_{2 R_{0} \Lambda_{t,+}^{(1)}}(0) \backslash B_{R}(0) \\
& =\left.8\left(\Lambda_{t,-}^{(1)}\right)^{2} \sum_{k=1}^{2} D_{y_{k}} D_{y_{l}} \ln h_{t}(y)\right|_{y=p_{t}^{(1)}} \int_{B_{R}(0)} \frac{b_{k}|z|^{2}}{\left(1+|z|^{2}\right)^{3}} \\
& +\frac{O\left(t^{2}\right)}{R}+O\left(t^{3} \ln R\right)+O\left(t^{4} R\right)+o\left(t^{2}\right),
\end{aligned}
$$

for some $\varepsilon \in(0,1)$. Therefore, we obtain from (3.81), (3.84), and (3.91) that 


$$
\begin{aligned}
& \text { (RHS) of (3.77) } \\
& =\left.8\left(\Lambda_{t,-}^{(1)}\right)^{2} \sum_{k=1}^{2} D_{y_{k}} D_{y_{l}} \ln h_{t}(y)\right|_{y=p_{t}^{(1)}}\left(\int_{\mathbb{R}^{2}} \frac{b_{k}|z|^{2}}{\left(1+|z|^{2}\right)^{3}} d z+O\left(R^{-2}\right)\right) \\
& \quad+\frac{O\left(t^{2}\right)}{R}+O\left(t^{3} R^{2}\right)+o\left(t^{2}\right) .
\end{aligned}
$$

Since $\operatorname{det}\left[\nabla^{2}\left(\ln h_{t}\left(p_{t}^{(1)}\right)\right)\right]=-16+O(t),(3.80)$ and (3.92) imply for $i=1,2$,

$$
\left|b_{i}\right|=O\left(\frac{1}{R}\right)+o(1) \text { for any large } \quad R \gg 1 .
$$

So we obtain $b_{1}=b_{2}=0$, and prove Lemma 3.8-(i).

(ii) By Lemma 3.3, Lemma 3.6-(v), and $b_{1}=b_{2}=0$, we have

$$
\widetilde{\zeta}_{t}(z)=\zeta_{t}\left(t \Lambda_{t,-}^{(1)} z+t p_{t}^{(1)}\right)-\frac{\int_{M} h e^{u_{t}^{(1)}-G_{t}} \zeta_{t} d v_{g}}{\int_{M} h e^{u_{t}^{(1)}-G_{t}} d v_{g}} \rightarrow 0 \text { in } C_{\mathrm{loc}}^{0}\left(\mathbb{R}^{2}\right)
$$

Together with Lemma 3.4-(ii), we obtain Lemma 3.8-(ii).

(iii) In view of Lemma 3.3, (3.25), and $b_{0}=b_{1}=b_{2}=0$, it is easy to see that Lemma 3.8-(iii) holds.

Now we are going to complete the proof of Theorem 1.2.

Proof of Theorem 1.2. Let $x_{t}^{*}$ be a maximum point of $\zeta_{t}$. So we have

$$
\left|\zeta_{t}\left(x_{t}^{*}\right)\right|=1
$$

Then from Lemma 3.4, we have

$$
\lim _{t \rightarrow 0} x_{t}^{*}=0
$$

Moreover, by Lemma 3.6-(iii) and Lemma 3.8, we see that

$$
\Lambda_{t,-}^{(1)} t \ll s_{t}:=\left|x_{t}^{*}-t p_{t}^{(1)}\right| \leq \frac{t R_{0}}{2} .
$$

Let $\hat{\zeta}_{t}(\xi)=\zeta_{t}\left(s_{t} \xi+t p_{t}^{(1)}\right)=\widetilde{\zeta}_{t}\left(\Lambda_{t,+}^{(1)} \frac{s_{t}}{t} \xi\right)+\frac{\int_{M} h e^{u_{t}^{(1)}-G_{t}} \zeta_{t} d v_{g}}{\int_{M} h e^{u_{t}^{(1)}-G_{t}} d v_{g}}$. By (3.25), $\hat{\zeta}_{t}$ satisfies

$$
0=\Delta \hat{\zeta}_{t}+O\left(\left(\Lambda_{t,+}^{(1)}\right)^{2} \frac{s_{t}^{2}}{t^{2}}\right) \frac{\left(1+\frac{s_{t}^{2}}{t^{2}}|\xi|^{2}\right)}{\left(1+\left(\Lambda_{t,+}^{(1)}\right)^{2} \frac{s_{t}^{2}}{t^{2}}|\xi|^{2}\right)^{2}} \quad \text { for } \quad|\xi| \leq \frac{r_{0}}{s_{t}}
$$

By (3.94), we have 


$$
\left|\hat{\zeta}_{t}\left(\frac{x_{t}^{*}-t p_{t}^{(1)}}{s_{t}}\right)\right|=\left|\zeta_{t}\left(x_{t}^{*}\right)\right|=1
$$

By (3.96) and $\left|\hat{\zeta}_{t}\right| \leq 1$, we see that $\hat{\zeta}_{t} \rightarrow \hat{\zeta}_{0}$ in any compact subset of $\mathbb{R}^{2} \backslash\{0\}$, where $\hat{\zeta}_{0}$ satisfies $\Delta \hat{\zeta}_{0}=0$ in $\mathbb{R}^{2} \backslash\{0\}$. Since $\left|\hat{\zeta}_{0}\right| \leq 1$, we have $\Delta \hat{\zeta}_{0}=0$ in $\mathbb{R}^{2}$. So $\hat{\zeta}_{0}$ is a constant. From $\frac{\left|x_{t}^{*}-t p_{t}^{(1)}\right|}{s_{t}}=1$ and $(3.98)$, we have $\hat{\zeta}_{0} \equiv 1$ or $\hat{\zeta}_{0} \equiv-1$. So we have

$$
\left|\zeta_{t}(x)\right| \geq \frac{1}{2} \quad \text { if } \quad \frac{s_{t}}{2} \leq\left|x-t p_{t}^{(1)}\right| \leq s_{t}
$$

By Lemma 3.5-(ii), Lemma 3.8, and Lemma 3.7, we see that if $\frac{s_{t}}{2} \leq\left|x-t p_{t}^{(1)}\right| \leq s_{t}$, then

$$
\zeta_{t}(x)=O(\ln t) \int_{B_{2 R_{0} \Lambda_{t,+}^{(1)}(0)}} \Delta \widetilde{\zeta}_{t} d z+o(1)=o(1) \quad \text { as } \quad t \rightarrow 0
$$

which contradicts (3.99). So we complete the proof of Theorem 1.2.

\section{Acknowledgments}

The authors wish to thank an anonymous referee very much for careful reading and valuable comments. The second author was supported by the National Research Foundation of Korea (NRF) grant funded by the Korea government (MSIT) (No. NRF-2018R1C1B6003403).

\section{References}

[1] S. Baraket, F. Pacard, Construction of singular limits for a semilinear elliptic equation in dimension 2, Calc. Var. Partial Differential Equations 6 (1) (1998) 1-38.

[2] D. Bartolucci, C.C. Chen, C.S. Lin, G. Tarantello, Profile of blow-up solutions to mean field equations with singular data, Comm. Partial Differential Equations 29 (7-8) (2004) 1241-1265.

[3] D. Bartolucci, A. Jevnikar, Y. Lee, W. Yang, Uniqueness of bubbling solutions of mean field equations, preprint.

[4] D. Bartolucci, G. Tarantello, Liouville type equations with singular data and their applications to periodic multivortices for the electroweak theory, Comm. Math. Phys. 229 (1) (2002) 3-47.

[5] D. Bartolucci, G. Tarantello, Asymptotic blow-up analysis for singular Liouville type equations with applications, J. Differential Equations 262 (7) (2017) 3887-3931.

[6] L. Battaglia, A. Malchiodi, Existence and non-existence results for the $S U(3)$ singular Toda system on compact surfaces, J. Funct. Anal. 270 (10) (2016) 3750-3807.

[7] H. Brezis, F. Merle, Uniform estimates and blow-up behavior for solutions of $-\Delta u=V(x) e^{u}$ in two dimensions, Comm. Partial Differential Equations 16 (1991) 1223-1254.

[8] C.L. Chai, C.S. Lin, C.L. Wang, Mean field equations, hyperelliptic curves and modular forms: I, Cambridge J. Math. 3 (1-2) (2015) 127-274.

[9] H. Chan, C.C. Fu, C.S. Lin, Non-topological multi-vortex solutions to the self-dual Chern-SimonsHiggs equation, Comm. Math. Phys. 231 (2) (2002) 189-221.

[10] W.X. Chen, C. Li, Classification of solutions of some nonlinear elliptic equations, Duke Math. J. 63 (3) (1991) 615-622.

[11] C.C. Chen, C.S. Lin, Sharp estimates for solutions of multi-bubbles in compact Riemann surface, Comm. Pure Appl. Math. 55 (2002) 728-771. 
[12] C.C. Chen, C.S. Lin, Topological degree for a mean field equation on Riemann surfaces, Comm. Pure Appl. Math. 56 (2003) 1667-1727.

[13] C.C. Chen, C.S. Lin, Mean field equations of Liouville type with singular data: shaper estimates, Discrete Contin. Dyn. Syst. 28 (3) (2010) 1237-1272.

[14] C.C. Chen, C.S. Lin, Mean field equation of Liouville type with singular data: topological degree, Comm. Pure Appl. Math. 68 (6) (2015) 887-947.

[15] K. Choe, N. Kim, Blow-up solutions of the self-dual Chern-Simons-Higgs vortex equation, Ann. Inst. H. Poincaré Anal. Non Linéaire 25 (2) (2008) 313-338.

[16] K. Choe, N. Kim, C.-S. Lin, Existence of self-dual non-topological solutions in the Chern-Simons Higgs model, Ann. Inst. H. Poincaré Anal. Non Linéaire 28 (2011) 837-852.

[17] J. Dolbeault, M.J. Esteban, G. Tarantello, Multiplicity results for the assigned Gauss curvature problem in $\mathbb{R}^{2}$, Nonlinear Anal. 70 (8) (2009) 2870-2881.

[18] A. Eremenko, Metrics of positive curvature with conic singularities on the sphere, Proc. Amer. Math. Soc. 132 (11) (2004) 3349-3355.

[19] P. Esposito, M. Grossi, A. Pistoia, On the existence of blowing-up solutions for a mean field equation, Ann. Inst. H. Poincaré Anal. Non Linéaire 22 (2) (2005) 227-257.

[20] P. Figueroa, Singular limits for Liouville-type equations on the flat two-torus, Calc. Var. Partial Differential Equations 49 (1-2) (2014) 613-647.

[21] C.F. Gui, A. Moradifam, The Sphere Covering Inequality and Its Applications, preprint.

[22] R. Jackiw, E.J. Weinberg, Self-dual Chern-Simons vortices, Phys. Rev. Lett. 64 (1990) 2234-2237.

[23] Y. Lee, C.S. Lin, G. Tarantello, W. Yang, Sharp estimates for the solutions with collapsing singularity, preprint.

[24] Y. Lee, C.S. Lin, J.C. Wei, W. Yang, Degree counting and Shadow system for Toda system of rank two: one bubbling, preprint.

[25] Y. Lee, C.S. Lin, W. Yang, Existence of bubbling solutions without mass concentration, preprint.

[26] Y. Lee, C.S. Lin, W. Yang, L. Zhang, Degree counting for Toda system with simple singularity: one point blow up, preprint.

[27] Y.Y. Li, I. Shafrir, Blow-up analysis for solutions of $-\Delta u=V(x) e^{u}$ in dimension two, Indiana Univ. Math. J. 43 (1994) 1255-1270.

[28] C.S. Lin, Uniqueness of solutions to the mean field equations for the spherical Onsager vortex, Arch. Ration. Mech. Anal. 153 (2) (2000) 153-176.

[29] C.S. Lin, An expository survey on the recent development of mean field equations, Discrete Contin. Dyn. Syst. 19 (2) (2007) 387-410.

[30] C.S. Lin, G. Tarantello, When "blow-up" does not imply "concentration": a detour from BrezisMerle's result, C. R. Math. Acad. Sci. Paris 354 (5) (2016) 493-498.

[31] C.S. Lin, C.L. Wang, Elliptic functions, Green functions and the mean field equations on tori, Ann. of Math. (2) 172 (2) (2010) 911-954.

[32] C.S. Lin, C.L. Wang, On the minimality of extra critical points of Green functions on flat tori, Int. Math. Res. Not. 176 (2016) 1-18.

[33] C.S. Lin, S.S. Yan, Existence of bubbling solutions for Chern-Simons model on a torus, Arch. Ration. Mech. Anal. 207 (2) (2013) 353-392.

[34] C.S. Lin, S.S. Yan, On the Chern-Simons-Higgs equation: Part II, local uniqueness and exact number of solutions, preprint.

[35] A. Malchiodi, Morse theory and a scalar field equation on compact surfaces, Adv. Differential Equations 13 (11-12) (2008) 1109-1129.

[36] A. Malchiodi, C.B. Ndiaye, Some existence results for the Toda system on closed surfaces, Atti Accad. Naz. Lincei Cl. Sci. Fis. Mat. Natur. Rend. Lincei (9) Mat. Appl. 18 (4) (2007) 391-412.

[37] A. Malchiodi, D. Ruiz, On the Leray-Schauder degree of the Toda system on compact surfaces, Proc. Amer. Math. Soc. 143 (7) (2015) 2985-2990.

[38] L. Nirenberg, Topics in Nonlinear Functional Analysis, Courant Institute of Mathematical Sciences, New York University, New York, 1974, viii+259 pp.

[39] M. Nolasco, G. Tarantello, Double vortex condensates in the Chern-Simons-Higgs theory, Calc. Var. Partial Differential Equations 9 (1) (1999) 31-94.

[40] G. Tarantello, Selfdual Gauge Field Vortices. An analytical approach, Progr. Nonlinear Differential Equations Appl., Birkhauser Boston, Inc., Boston, 2008.

[41] G. Tarantello, Analytical, Geometrical and Topological aspects of mean field equations on surfaces, Discr. Cont. Dyn. Syst. 28 (3) (2010) 931-973.

[42] M. Troyanov, Metrics of constant curvature on a sphere with two conical singularities, in: Differential Geometry, Peniscola, 1988, in: Lecture Notes in Math., vol. 1410, Springer, Berlin, 1989, pp. 296-306. 
[43] Y. Yang, The relativistic non-Abelian Chern-Simons equations, Comm. Math. Phys. 186 (1997) 199-218.

[44] Y. Yang, Solitons in Field Theory and Nonlinear Analysis, Springer Monogr. Math., Springer, New York, 2001.

[45] L. Zhang, Asymptotic behavior of blowup solutions for elliptic equations with exponential nonlinearity and singular data, Commun. Contemp. Math. 11 (2009) 395-411. 